HIAS-E-49

\title{
Globalisation and Inequality in a Dynamic Economy: An Axiomatic Analysis of Unequal Exchange
}

Roberto Veneziani

School of Economics and Finance, Queen Mary University of London, Mile End Road, London E1 4NS, UK

Naoki Yoshihara

University of Massachusetts Amherst, Crotty Hall, 412 North Pleasant Street, Amherst, MA 01002, USA The Institute of Economic Research, Hitotsubashi University, Naka 2-1, Kuni- tachi, Tokyo 186-8603, Japan School of Management, Kochi University of Technology, Tosayamada, Kami-city, Kochi 782-8502, Japan

February 2017

Hitotsubashi Institute for Advanced Study, Hitotsubashi University 2-1, Naka, Kunitachi, Tokyo 186-8601, Japan

tel:+81 425808604 http://hias.ad.hit-u.ac.jp/

HIAS discussion papers can be downloaded without charge from: http://hdl.handle.net/10086/27202

https://ideas.repec.org/s/hit/hiasdp.html

All rights reserved. 


\title{
Globalisation and Inequality in a Dynamic Economy:
}

\section{An Axiomatic Analysis of Unequal Exchange ${ }^{1}$}

\author{
Roberto Veneziani $^{2} \quad$ Naoki Yoshihara ${ }^{3}$
}

February 24, 2017

\footnotetext{
${ }^{1}$ Paper prepared for the special issue in honour of John Roemer. We thank Jon Cogliano, Amitava Dutt, Gary Dymski, Peter H. Matthews, Rajiv Sethi, Gil Skillman, Peter Skott, the Editors of this journal, two anonymous referees, and audiences in London, New York, Chicago, Amherst, and Sendai, for useful comments and suggestions. The usual disclaimer applies.

${ }^{2}$ School of Economics and Finance, Queen Mary University of London, Mile End Road, London E1 4NS, UK. E-mail: r.veneziani@qmul.ac.uk

${ }^{3}$ (Corresponding author) Department of Economics, University of Massachusetts Amherst, Crotty Hall, 412 North Pleasant Street, Amherst, MA 01003, USA; The Institute of Economic Research, Hitotsubashi University, Kunitachi, Tokyo 186-0004 Japan; and School of Management, Kochi University of Technology, Kochi 782-8502, Japan. E-mail: nyoshihara@econs.umass.edu
} 


\begin{abstract}
An axiomatic analysis of the concept of unequal exchange (UE) between countries is developed in a dynamic general equilibrium model that generalises Roemer's [22] economy with a global capital market. The class of UE definitions that satisfy three fundamental properties including a correspondence between wealth, class and UE exploitation status - is completely characterised. It is shown that this class is nonempty and a definition of UE exploitation between countries is proposed, which is theoretically robust and firmly anchored to empirically observable data. The full class and UE exploitation structure of the international economy is derived in equilibrium.
\end{abstract}

JEL classification: D63; F02; B51.

Keywords: Exploitation, classes, unequal exchange, international economy. 


\section{Introduction}

Egalitarians - and more specifically, socialists - have long questioned the structure of international relations, pointing in particular at the large inequalities in income and standard of living among countries. An influential analysis within this tradition has been developed by the so-called dependence school, according to which such inequalities are the product of exploitative relations between rich and poor nations. ${ }^{1}$ In his classic work, for example, Emmanuel [9] has argued that the core-periphery structure of international relations generates an unequal exchange (UE) between rich and poor nations. According to Emmanuel, given institutionalised wage differentials between developed and less developed nations, capital mobility across borders and the international trade of commodities cause a transfer of surplus labour from poor nations with low capital-labour ratios to wealthy nations with high capital-labour ratios, which results in the impoverishment of the former to the advantage of the latter.

UE theory has generated substantial controversy on both empirical and theoretical grounds. Empirically, some studies emphasise the positive effects of international exchanges and provide evidence suggesting that the "movement toward free trade may actually have just the opposite effect [than predicted by UE theory], leading to a reduction in income disparity among countries" (Ben-David [4], p.653). UE theory is deemed theoretically doubtful, because it is based on the comparison between the amounts of labour embodied in the goods traded, and the notion of labour embodied is widely considered to be metaphysical and at best - of very limited validity, as it can only be defined in rather special cases. ${ }^{2}$ In this paper, we examine the theoretical foundations of UE theory in order to determine whether a general, consistent, and empirically grounded definition of UE can be identified. ${ }^{3}$

The starting point of our investigation is a seminal contribution by Roemer [22], which

\footnotetext{
${ }^{1}$ The literature is too vast for a comprehensive list of references. For a discussion of the classic contributions, we refer the reader to the excellent reviews by Bacha [3] and Griffin and Gurley [13].

${ }^{2} \mathrm{UE}$ theory is also criticised because it contradicts the principle of comparative advantage, according to which profit equalisation and capital flows from rich to poor countries have growth-inducing and inequalityreducing effects. See, for example, the debate between Paul Samuelson and Arghiri Emmanuel in The Journal of International Economics in 1978.

${ }^{3}$ Empirically, we simply note that recent studies have provided evidence supporting the idea that international inequalities have indeed increased. See, for example, Slaughter [26].
} 
provides an elegant analysis of UE between countries, in a static model with revenuemaximising countries and a Leontief technology (see also Roemer [21]). Roemer [22] departs from the standard UE approach in two key respects. First, he adopts a rigorous definition of UE exploitation that is unrelated to the labour theory of value and that does not presume global markets to be non-competitive. Second, in order to analyse the normative foundations of UE theory, he focuses on a model in which "all differences between countries [are eliminated] save one: the capital-labor ratio" (Roemer [22], p.35). This allows him to show that class relations and UE exploitation emerge in the international economy, even assuming perfectly competitive global commodity and credit markets, and even if all countries have access to the same technology and share the same preferences. In equilibrium, the global economy is characterised both by mutual gains from trade and by asymmetric international relations because the economic development of less developed countries is crucially dependent on capital exports from developed countries, and surplus is transferred from the former to the latter via international capital markets. "Unequal exchange does not preempt mutual gains from trade" (ibid.). ${ }^{4}$ Major heterogeneities in institutions, technology or preferences and market imperfections are unessential for these results: unequal and asymmetric relations between countries "can be entirely explained by differential capital-labor ratios across countries" (Roemer [22], p.34).

As insightful as Roemer's [21, 22] contributions are, it is still an open question whether his key insights are robust. It is not clear, for example, whether Roemer's conclusions hold under more general assumptions concerning preferences and technology, and outside of static models. In later contributions, Roemer himself has raised doubts on the generality of UE theory, ${ }^{5}$ and on the possibility of identifying a rigorous definition that captures the fundamental normative intuitions of UE exploitation.

In this paper, we investigate the robustness of UE theory and extend Roemer's [21, 22] analysis by adopting a more general formal framework and a different methodological approach. Formally, instead of restricting attention to static, one-period economies, we set up

\footnotetext{
${ }^{4}$ This insight is compatible with the classical Marxian theory of exploitation, as Marx ([15], chapter 20, (e)) notes that "a richer country exploits a poorer one, even when the latter benefits from the exchange."

${ }^{5}$ For example, Roemer [23]. See Veneziani [28] for a thorough discussion.
} 
a dynamic general equilibrium model of the global economy in which intertemporal decisions are explicitly considered and the concept of UE exploitation is analysed at general dynamic equilibria. Further, the model incorporates more general assumptions on preferences - by assuming that countries' welfare depends both on consumption and on leisure - and on technology - by allowing for convex production sets.

Methodologically, instead of focusing on a specific definition of UE exploitation, we adopt the axiomatic approach pioneered by Yoshihara and Veneziani [33] and significantly extend it by considering different properties, and by requiring our axioms to hold at general dynamic equilibria. We examine three fundamental properties. The first one, called Labour Exploitation $(\mathbf{L E})$, is a domain axiom that captures some basic intuitions defining the core of UE theory that all admissible definitions should satisfy. It is a dynamic generalisation of similar properties formulated in the literature (see, e.g., [31, 29, 30]), and it reduces to the latter in the special case of a static, one-period economy. Intuitively, according to LE, exploitative international relations are characterised by systematic differences between the labour performed by agents in a country and the amount of labour 'contained' in some reference commodity bundles that capture their consumption possibilities. ${ }^{6}$

The other two properties, called the Class-Exploitation Correspondence Principle (CECP) and the Wealth-Exploitation Correspondence Principle (WECP) capture axiomatically two intuitions of UE theory originally introduced by Roemer [21, 22], who proved them to hold in certain economies, under specific UE definitions. According to $\mathbf{C E C P}$, a correspondence should exist between a country's position in the global capital market and its exploitation status: in equilibrium, nations that optimise by lending capital abroad should emerge as UE exploiters, whereas nations that optimise by borrowing capital should be UE exploited. CECP has been first formulated axiomatically by Yoshihara [31] in a static context; we extend it here to hold at general dynamic equilibria. WECP is a novel axiom in the literature: it states that the exploitation status of countries in the international arena should be determined by their level of development (proxied by the value of their productive endowments).

\footnotetext{
${ }^{6}$ A rigorous statement of all three axioms is in section 4. $\mathbf{L E}$ is conceptually related to the classic theories of unequal exchange (Emmanuel [9]) and underdevelopment (Amin [2], Frank [11]).
} 
We completely characterise the class of UE definitions that satisfy all three axioms at the general dynamic equilibria of the international economy. Then, we prove that this class is nonempty: a definition recently proposed by Yoshihara and Veneziani [31, 33, 29] based on the 'New Interpretation' (Duménil [6, 7]; Foley [12]; Duménil et al [8]) satisfies all three properties. Contrary to the received view, a rigorous and logically consistent definition of unequal, exploitative exchange exists, which is firmly anchored to empirically observable data and extends the key insights of UE theory to general, dynamic international economies.

Our analysis here continues a research programme in axiomatic exploitation theory started in Yoshihara and Veneziani [33] (see also [31, 29, 30]) and significantly extends it to the dynamic context, and to deal with different intuitions of UE theory, formalised in a different set of axioms. We analyse general intertemporal economies because this allows us to evaluate the robustness of Roemer's [21, 22] key insights, and the generality of the 'New Interpretation', but also because they raise some important conceptual and formal issues in axiomatic UE theory. In fact, as shown below, in a dynamic context, the appropriate definition of UE exploitation depends on the normative relevance of agents' consumption and savings decisions, and this requires a number of adjustments in the conceptual framework, in the axiomatic system, and in the formal arguments.

To be sure, this paper does not fully answer the question of the normative relevance of UE and the wrongfulness of exploitative international relations. Yet the rigorous, axiomatic characterisation of a nonempty class of definitions that preserve some key insights of UE theory is a crucial first step in order to address that question. ${ }^{7}$

\section{The Model}

The economy consists of a set $\mathcal{N}=\{1, \ldots, N\}$ of countries, with generic element $\nu$, in which a sequence of nonoverlapping generations exist, each living for $T$ periods, ${ }^{8}$ and indexed by the

\footnotetext{
${ }^{7}$ We discuss some normative implications of UE exploitation theory in Veneziani [27, 28] and Yoshihara and Veneziani [33].

${ }^{8}$ We specify the framework in the case with a finite $T$ in order to highlight the similarity with Roemer's $[21,22]$ economies. However, the notation and definitions can be extended in a straightforward way to the case with one infinitely-lived generation, and all of our results hold both if $T$ is finite and if it is infinite.
} 
date of birth $k T, k=0,1,2, \ldots$ In every period $t$, countries consume $n$ produced commodities, and leisure. Technology is freely available to all countries: in every $t$, capitalists in each country can operate any activity in the production set $P \subseteq \mathbb{R}^{2 n+1}$, with generic element $\alpha=\left(-\alpha_{l},-\underline{\alpha}, \bar{\alpha}\right)$, where $\alpha_{l} \in \mathbb{R}_{+}$is the direct labour input; $\underline{\alpha} \in \mathbb{R}_{+}^{n}$ are the inputs of the $n$ goods; and $\bar{\alpha} \in \mathbb{R}_{+}^{n}$ are the outputs of the $n$ goods. The net output vector arising from $\alpha$ is denoted as $\widehat{\alpha} \equiv \bar{\alpha}-\underline{\alpha}$. Let $\mathbf{0}$ be the null vector. The set $P$ is assumed to be a closed convex cone containing the origin in $\mathbb{R}^{2 n+1}$, and to satisfy the following standard properties. ${ }^{9}$

Assumption 1 (A1). For all $\alpha \in P$, if $\bar{\alpha} \geq \mathbf{0}$ then $\alpha_{l}>0$ and $\underline{\alpha} \geq \mathbf{0 .}$

Assumption 2 (A2). For all $c \in \mathbb{R}_{+}^{n}$, there exists $\alpha \in P$ such that $\widehat{\alpha} \geqq c$.

Assumption 3 (A3). For all $\alpha \in P$, and for all $\left(-\underline{\alpha}^{\prime}, \bar{\alpha}^{\prime}\right) \in \mathbb{R}_{-}^{n} \times \mathbb{R}_{+}^{n}$, if $\left(-\underline{\alpha}^{\prime}, \bar{\alpha}^{\prime}\right) \leqq$ $(-\underline{\alpha}, \bar{\alpha})$ then $\left(-\alpha_{l},-\underline{\alpha}^{\prime}, \bar{\alpha}^{\prime}\right) \in P$.

A1 implies that labour and some capital are indispensable to produce any output; A2 states that any non-negative commodity bundle is producible as net output; A3 is a standard free disposal condition. The set of efficient production activities is $\partial P=\left\{\alpha \in P \mid \nexists \alpha^{\prime} \in P\right.$ such that $\left.\alpha^{\prime}>\alpha\right\}$

Commodities and capital can freely migrate across borders, while labour is immobile. In every $t,\left(p_{t}, r_{t}\right)$ is the $1 \times(n+1)$ international price vector, where $p_{t}$ denotes the prices of the $n$ commodities and $r_{t}$ is the interest rate that prevails in competitive capital markets. In order to focus on international inequalities, agents are assumed to be identical within each country; thus, the superscript $\nu$ denotes both a country and its representative agent.

Following Roemer [21, 22], we explicitly model the time structure of exchange and production. Each production period $t$ is divided into two stages: the capital market and the market for productive assets operate at the beginning of $t$, where goods are exchanged at the prices $p_{t-1}$ ruling at the end of $t-1$ /beginning of $t$. At the beginning of $t, \omega_{t}^{\nu}$ is the vector of productive assets owned by $\nu$, - where $\omega_{k T}^{\nu}$ denotes the endowments inherited when born in $k T$, - and the market value of $\nu$ 's endowments, $\nu$ 's wealth, is $W_{t}^{\nu}=p_{t-1} \omega_{t}^{\nu}$.

At the beginning of every $t$, each $\nu \in \mathcal{N}$ can borrow an amount $p_{t-1} \underline{\beta}_{t}^{\nu}$ on the international

\footnotetext{
${ }^{9}$ Vector inequalities: for all $x, y \in \mathbb{R}^{m}, x \geqq y$ if and only if $x_{i} \geqq y_{i}(i=1, \ldots, m) ; x \geq y$ if and only if $x \geqq y$ and $x \neq y ; x>y$ if and only if $x_{i}>y_{i}(i=1, \ldots, m)$.
} 
credit market to purchase $\underline{\beta}_{t}^{\nu}$ in order to operate production activity $\beta_{t}^{\nu}=\left(-\beta_{l t}^{\nu},-\underline{\beta}_{t}^{\nu}, \bar{\beta}_{t}^{\nu}\right) \in$ $P$. Otherwise, it can use its wealth $W_{t}^{\nu}$ either to purchase capital goods $\underline{\alpha}_{t}^{\nu}$ to operate activity $\alpha_{t}^{\nu}=\left(-\alpha_{l t}^{\nu},-\underline{\alpha}_{t}^{\nu}, \bar{\alpha}_{t}^{\nu}\right) \in P$; or to buy commodities $\delta_{t}^{\nu} \in \mathbb{R}_{+}^{n}$ to be stored and sold at the end of the period; or to lend capital $z_{t}^{\nu} \in \mathbb{R}_{+}$abroad.

Because production takes time, output is exchanged on the final goods market at the end of $t$, at end-of-period prices $p_{t}$. For each country $\nu \in \mathcal{N}$, proceedings from production are given by $p_{t}\left(\bar{\alpha}_{t}^{\nu}+\bar{\beta}_{t}^{\nu}\right)$ and the return to lending $z_{t}^{\nu}$ is $\left(1+r_{t}\right) z_{t}^{\nu}$, thus gross national income at the end of $t$ is $p_{t}\left(\bar{\alpha}_{t}^{\nu}+\bar{\beta}_{t}^{\nu}\right)+\left(1+r_{t}\right) z_{t}^{\nu}$ from which the rental cost of the borrowed capital $\left(1+r_{t}\right) p_{t-1} \underline{\beta}_{t}^{\nu}$ must be paid. The rest of $\nu$ 's income can be used to purchase consumption goods $c_{t}^{\nu} \in \mathbb{R}_{+}^{n}$ and to finance accumulation $\omega_{t+1}^{\nu} \in \mathbb{R}_{+}^{n}$.

Given production decisions $\left(\alpha_{t}^{\nu}, \beta_{t}^{\nu}\right)$, in every $t$, the total amount of labour performed by agents in $\nu \in \mathcal{N}$ is given by $\Lambda_{t}^{\nu}=\alpha_{l t}^{\nu}+\beta_{l t}^{\nu}$ and it cannot exceed the labour endowment, $L$, which is assumed to be the same for all countries without loss of generality. Therefore for each $\nu \in \mathcal{N}$, leisure enjoyed at $t$ is $l_{t}^{\nu}=L-\Lambda_{t}^{\nu}$, and we assume that country $\nu$ 's welfare at $t$ can be represented by a function $u: \mathbb{R}_{+}^{n} \times[0, L] \rightarrow \mathbb{R}_{+}: u\left(c_{t}, l_{t}\right)$ can be interpreted either as a standard utility function or as an objectivist index of well-being. ${ }^{10}$

In order to characterise the structure of international relations and the dynamic pattern of exploitation and classes, it is necessary to impose some structure on the function $u$. We assume that $u\left(c_{t}, l_{t}\right)=\phi\left(l_{t}\right)+v\left(c_{t}\right)$, where $v: \mathbb{R}_{+}^{n} \rightarrow \mathbb{R}$ and $\phi:[0, L] \rightarrow \mathbb{R}$ are strictly increasing and twice differentiable. Further, in order to avoid a number of unnecessary technicalities, we assume that $v$ is strictly quasi-concave and homogeneous of degree one, while $\phi$ is strictly concave with $\lim _{l \rightarrow L} \phi^{\prime}(l)=0$ and $\lim _{l \rightarrow 0} \phi^{\prime}(l)=\infty$.

These assumptions significantly generalise the canonical models of exploitation theory by Roemer [21, 22]. They are appropriate from a normative perspective (see, for example, Silvestre [25]), and standard in international economics - and specifically, in the literature on Heckscher-Ohlin models (see, for example, Chen [5]). The assumptions on $\phi$, for example, rule out implausible equilibria with countries performing zero labour, or enjoying no leisure

\footnotetext{
${ }^{10}$ For a discussion of subjective and objective principles, see Roemer and Veneziani [24] and, in the context of exploitation theory, Yoshihara and Veneziani [34].
} 
at all. It is worth stressing, however, that the restrictions on $u$ are imposed mostly for technical convenience, and the main results of this paper can be derived under more general assumptions, albeit at the cost of a significant increase in technicalities. ${ }^{11}$

Let $c^{\nu}=\left\{c_{t}^{\nu}\right\}_{t=k T}^{(k+1) T-1}$ be $\nu$ 's lifetime consumption plan; and likewise for $\alpha^{\nu}, \beta^{\nu}, z^{\nu}, \delta^{\nu}$, and $\Lambda^{\nu}$, and let $\omega^{\nu}=\left\{\omega_{t+1}^{\nu}\right\}_{t=k T}^{(k+1) T-1}$ be $\nu$ 's lifetime accumulation plan. Let $(\mathbf{p}, \mathbf{r})=$ $\left\{\left(p_{t}, r_{t}\right)\right\}_{t=k T}^{(k+1) T-1}$ be the path of international price vectors during the lifetime of a generation. Let $\xi^{\nu}=\left(\alpha^{\nu}, \beta^{\nu}, z^{\nu}, \delta^{\nu}, c^{\nu}, \omega^{\nu}\right)$ denote a generic intertemporal plan for $\nu$. Let $0<\rho \leqq 1$ be the time preference factor. Each $\nu$ is assumed to choose $\xi^{\nu}$ to maximise welfare subject to the constraint that in every $t,(1)$ gross national income is sufficient for consumption and accumulation; (2) wealth is sufficient for production and lending; (3) production activities are technologically feasible. Finally, (4) we follow Roemer [21, 22] and assume that each agent optimises subject to the requirement that he does "not run down the value of his assets, which is the reproducibility requirement" (Roemer [21], p.63). Unlike in Roemer's static economies, however, the reproducibility condition (4) does not hold in every period. In any given $t$, agents can use their wealth to finance consumption but every generation $k$ is constrained to bequeath at the end of its life at least as much wealth as they inherited. Formally, given $(\mathbf{p}, \mathbf{r})$, each $\nu$ solves programme $M P^{\nu} \cdot{ }^{12}$

$$
M P^{\nu}: V\left(\omega_{k T}^{\nu}\right)=\max _{\xi^{\nu}} \sum_{t=k T}^{(k+1) T-1} \rho^{t}\left[\phi\left(L-\Lambda_{t}^{\nu}\right)+v\left(c_{t}^{\nu}\right)\right]
$$

subject to: for every $t=k T, \ldots,(k+1) T-1$,

$$
\begin{aligned}
p_{t} \bar{\alpha}_{t}^{\nu}+\left[p_{t} \bar{\beta}_{t}^{\nu}-\left(1+r_{t}\right) p_{t-1} \underline{\beta}_{t}^{\nu}\right]+\left(1+r_{t}\right) z_{t}^{\nu}+p_{t} \delta_{t}^{\nu} & =p_{t} c_{t}^{\nu}+p_{t} \omega_{t+1}^{\nu}, \\
p_{t-1}\left(\underline{\alpha}_{t}^{\nu}+\delta_{t}^{\nu}\right)+z_{t}^{\nu} & =p_{t-1} \omega_{t}^{\nu}, \\
\alpha_{t}^{\nu}, \beta_{t}^{\nu} & \in P, \Lambda_{t}^{\nu} \leqq L, \\
p_{(k+1) T-1} \omega_{(k+1) T}^{\nu} & \geqq p_{(k+1) T-1} \omega_{k T}^{\nu} .
\end{aligned}
$$

\footnotetext{
${ }^{11}$ For example, it is possible to allow for heterogeneous preferences over consumption goods with $u^{\nu}\left(c_{t}^{\nu}, l_{t}^{\nu}\right)=\phi\left(L-\Lambda_{t}^{\nu}\right)+v^{\nu}\left(c_{t}^{\nu}\right)$; a weakly concave $\phi ; v$ being homogeneous of degree $k<1$; and so on.

${ }^{12}$ Constraints (1)-(2) are written as equalities without loss of generality, given the monotonicity of $u$.
} 
$M P^{\nu}$ is a suitable way of modelling country $\nu$ 's decision problem, given the representativeagent assumption, and it generalises Roemer's [21, 22] static models in which countries maximise national income.

In order to capture the role of financial markets in exploitative international relations, only short-term credit contracts are considered as in Roemer [21, 22]: within each period, countries can operate on the international capital market to finance their production plans, but contracts do not extend over time and credit plays a limited role in fostering accumulation. Consumption, debt, and savings must be financed out of current revenue. Due to the possibility of saving, and noting that net savings are allowed to be negative, however, Roemer's $[21,22]$ static models are generalised by allowing for intertemporal trade-offs within a country, consistently with a dynamic setting in which agents live for more than one period.

For all $\nu \in \mathcal{N}$, let $\mathcal{O}^{\nu}(\mathbf{p}, \mathbf{r})$ be the set of vectors $\xi^{\nu}$ that solve $M P^{\nu}$ at prices $(\mathbf{p}, \mathbf{r})$. Let $\Omega_{k T}=\left(\omega_{k T}^{1}, \omega_{k T}^{2}, \ldots, \omega_{k T}^{N}\right)$. Let $E\left(P, \mathcal{N}, u, \rho, \Omega_{k T}\right)$, or as a shorthand notation $E\left(\Omega_{k T}\right)$, denote the international economy with technology $P$, countries $\mathcal{N}$, welfare function $u$ with discount factor $\rho$, and productive endowments $\Omega_{k T}$. Let $c_{t}=\sum_{\nu \in \mathcal{N}} c_{t}^{\nu}$; and likewise for all other variables. For the sake of simplicity, let "for all $t$ " stand for "for all $t=k T, \ldots,(k+1) T-1$ ". Following Roemer [21, 22], the equilibrium concept can now be defined..$^{13}$

Definition 1: A reproducible solution (RS) for $E\left(\Omega_{k T}\right)$ is a price vector $(\mathbf{p}, \mathbf{r})$ and an associated profile of actions $\left(\xi^{\nu}\right)_{\nu \in \mathcal{N}}$ such that:

(i) $\xi^{\nu} \in \mathcal{O}^{\nu}(\mathbf{p}, \mathbf{r})$ for all $\nu \in \mathcal{N}$;

(ii) $\bar{\alpha}_{t}+\bar{\beta}_{t}+\delta_{t} \geqq c_{t}+\omega_{t+1}$ with $\bar{\alpha}_{t}+\bar{\beta}_{t} \geq \mathbf{0}$ for all $t$;

(iii) $\underline{\alpha}_{t}+\underline{\beta}_{t}+\delta_{t} \leqq \omega_{t}$ for all $t$;

(iv) $p_{t-1} \underline{\beta}_{t}=z_{t}$ for all $t$;

(v) $\omega_{(k+1) T} \geqq \omega_{k T}$.

In other words, at a RS, (i) every country optimises. Conditions (ii) and (iii) are standard excess demand conditions: in the markets for final goods and capital goods, respectively, aggregate demand should not exceed aggregate supply in any period. Condition (ii) also

\footnotetext{
${ }^{13}$ The existence of a reproducible solution is proved in the Addendum.
} 
requires the RS to be non-trivial in that some production takes place in every period. Condition (iv) requires that the international credit market clears in every period. Finally, the reproducibility condition (v) is a generalisation of analogous equilibrium conditions imposed by Roemer [20, 21, 22]: it requires that every generation leaves to the following at least as many resources as they inherited. As Roemer ([20], p.507) has argued, the notion of reproducibility is one of the distinctive features of the concept of equilibrium in the formal literature on exploitation theory (compared to a more standard concept of general equilibrium). It is also a standard condition in Ramsey-type growth models with a finite horizon (see, e.g., Morishima's [16] classic model) and it is quite natural given that countries - rather than individuals - are the focus of analysis. For, although each generation dies, the country itself lives on, and so its capital stock should not be depleted.

In the rest of this section, we derive some preliminary results that describe the characteristics of the equilibria of the international economy. First, the strict monotonicity of $v$ implies that at any RS, it must be $p_{t}>\mathbf{0}$ for all $t$. Next, given $(\mathbf{p}, \mathbf{r})$, at any $t$, let $w_{t}^{\max }=\max _{\alpha \in P} \frac{p_{t} \bar{\alpha}-\left(1+r_{t}\right) p_{t-1} \underline{\underline{\alpha}}}{\alpha_{l}}$ : by the assumptions on $P, w_{t}^{\max }$ is well-defined. Hence let $P_{t}^{w}(\mathbf{p}, \mathbf{r})=\left\{\alpha \in P \mid w_{t}^{\max }=\frac{p_{t} \bar{\alpha}-\left(1+r_{t}\right) p_{t-1} \underline{\underline{\alpha}}}{\alpha_{l}}\right\}$. Proposition 1 proves that only processes with the highest return to labour are activated and, as is well-known in international economics, even without an international labour market, wages are equalised in all countries at all $t$.

Proposition 1: Let $\left((\mathbf{p}, \mathbf{r}),\left(\xi^{\nu}\right)_{\nu \in \mathcal{N}}\right)$ be a RS for $E\left(\Omega_{k T}\right)$. Then $w_{t}^{\max }>0$ for all $t$, and $\alpha_{t}^{\nu}, \beta_{t}^{\nu} \in P_{t}^{w}(\mathbf{p}, \mathbf{r})$ for all $\nu$ and all $t$.

Proof: 1 . Suppose, contrary to the statement, that $w_{t}^{\max } \leqq 0$ for some $t$. Then $1+r_{t}>0$ and at the solution to $M P^{\nu}$, it must be $\bar{\alpha}_{t}^{\nu}=\bar{\beta}_{t}^{\nu}=\mathbf{0}$ for all $\nu$. To see this, note that, since $p_{t}>\mathbf{0}$, all $t$, if either $\bar{\alpha}_{t}^{\nu} \geq \mathbf{0}$ or $\bar{\beta}_{t}^{\nu} \geq \mathbf{0}$ for some $\nu$, then by A1 agent $\nu$ can reduce labour performed and increase revenues by setting $\alpha_{t}^{\prime \nu}=\beta_{t}^{\prime \nu}=\mathbf{0}$ and $z_{t}^{\prime}=z_{t}+p_{t-1} \underline{\alpha}^{\nu}$. Therefore $\bar{\alpha}_{t}=\bar{\beta}_{t}=\mathbf{0}$ which contradicts Definition $1(\mathrm{~b})$.

2. The second part of the statement follows immediately from $M P^{\nu}$.

At any RS, $1+r_{t} \geqq \max _{i} \frac{p_{i t}}{p_{i t-1}}$ must also hold at all $t$. For if there were some good $i$ such that $\frac{p_{i t}}{p_{i t-1}}>1+r_{t}$, at some $t$, then noting that by Proposition $1 w_{t}^{\max }>0$, at the solution 
to $M P^{\nu}$ the gross revenue would have the form of $w_{t}^{\max } \beta_{l t}^{\nu}+p_{t} \delta_{t}^{\nu}$ with $p_{t-1} \delta_{t}^{\nu}=p_{t-1} \omega_{t}^{\nu}$ and $\beta_{t}^{\nu} \in P_{t}^{w}(\mathbf{p}, \mathbf{r})$, where $\beta_{l t}^{\nu}>0$ follows from the assumptions of $\phi$. Then, $\alpha_{t}^{\nu}=\mathbf{0}, z_{t}^{\nu}=0$, and $p_{t-1} \underline{\beta}_{t}^{\nu}>0$ by $p_{t}>\mathbf{0}$ and A1, all $\nu \in \mathcal{N}$. This contradicts Definition 1(iv).

Lemma 1 proves a useful property of the set of solutions of $M P^{\nu}$.

Lemma 1: Let $(\mathbf{p}, \mathbf{r})$ be a price vector such that $w_{t}^{\max }>0$ and $\left(1+r_{t}\right) \geqq \max _{i} \frac{p_{i t}}{p_{i t-1}}$ for all t. For all $\nu \in \mathcal{N}$, if $\left(\alpha^{\nu}, \beta^{\nu}, z^{\nu}, \delta^{\nu}, c^{\nu}, \omega^{\nu}\right)$ solves $M P^{\nu}$, then $\left(\alpha^{\prime \nu}, \beta^{\prime \nu}, z^{\prime \nu}, \delta^{\nu}, c^{\nu}, \omega^{\nu}\right)$ also solves $M P^{\nu}$ whenever $\alpha_{t}^{\prime \nu}+\beta_{t}^{\nu \nu}=\alpha_{t}^{\nu}+\beta_{t}^{\nu}, z_{t}^{\prime \nu}+p_{t-1} \underline{\alpha}_{t}^{\nu \nu}=z_{t}^{\nu}+p_{t-1} \underline{\alpha}_{t}^{\nu}, \alpha_{t}^{\prime \nu}, \beta_{t}^{\nu \nu} \in P$, and $z_{t}^{\prime \nu} \geqq 0$, all $t$.

Proof: By construction, the constraints of $M P^{\nu}$ are all satisfied. Furthermore, $\beta_{l t}^{\prime \nu}+\alpha_{l t}^{\prime \nu}=$ $\beta_{l t}^{\nu}+\alpha_{l t}^{\nu}$, at all $t$ and $c^{\prime \nu}=c^{\nu}$. Hence $\left(\alpha^{\prime \nu}, \beta^{\prime \nu}, z^{\prime \nu}, \delta^{\nu}, c^{\nu}, \omega^{\nu}\right)$ yields the same welfare.

By Lemma 1, and the convexity of $P$, we can consider solutions of $M P^{\nu}$ with $\alpha^{\nu}=\mathbf{0}$, without loss of generality.

For all $k$, at the solution to $M P^{\nu}, p_{(k+1) T-1} \omega_{(k+1) T}^{\nu}=p_{(k+1) T-1} \omega_{k T}^{\nu}$, all $\nu$. Hence, at a RS, $\omega_{(k+1) T}=\omega_{k T}$ since $p_{(k+1) T-1}>\mathbf{0}$. Moreover, $\omega_{(k+1) T}^{\nu}=\omega_{k T}^{\nu}$ is feasible and optimal for all $\nu$. Therefore, without loss of generality, we can focus on solutions with $\omega_{(k+1) T}^{\nu}=\omega_{k T}^{\nu}$. Hence, if $\left((\mathbf{p}, \mathbf{r}),\left(\xi^{\nu}\right)_{\nu \in \mathcal{N}}\right)$ is a RS for $E\left(\Omega_{k T}\right)$, then it is also a RS for $E\left(\Omega_{(k+1) T}\right)$, and in what follows generation $k=0$ can be considered without loss of generality.

A subset of equilibria of particular interest are those where agents optimise at an interior solution. Thus:

Definition 2: An interior $R S$ (IRS) for $E\left(\Omega_{k T}\right)$ is a $\mathrm{RS}$ such that for all $\nu, \xi^{\nu} \in \mathcal{O}^{\nu}(\mathbf{p}, \mathbf{r})$ is an interior solution to $M P^{\nu}$ with $c_{t}^{\nu}>\mathbf{0}$ for all $t$.

The next result proves a necessary condition for an IRS.

Lemma 2: Let $\left((\mathbf{p}, \mathbf{r}),\left(\xi^{\nu}\right)_{\nu \in \mathcal{N}}\right)$ be an IRS for $E\left(\Omega_{0}\right)$. Then for all $\nu \in \mathcal{N}, \Lambda^{\nu}=\Lambda^{*}=$ $\left\{\Lambda_{t}^{*}\right\}_{t=k T}^{(k+1) T-1}$ where $\frac{\phi^{\prime}\left(L-\Lambda_{t}^{*}\right)}{w_{t}^{\max }}=\rho\left(1+r_{t+1}\right) \frac{\phi^{\prime}\left(L-\Lambda_{t+1}^{*}\right)}{w_{t+1}^{\max }}$, for all $t$.

Proof: 1. By Proposition 1, for all $\nu \in \mathcal{N}$, at the solution to $M P^{\nu}$, it must be $\left(1+r_{t}\right) p_{t-1} \omega_{t}^{\nu}+$ $w_{t}^{\max } \Lambda_{t}^{\nu}=p_{t} c_{t}+p_{t} \omega_{t+1}^{\nu}$, all $t$. Then it is immediate to prove that, at an interior so- 
lution $\xi^{\nu} \in \mathcal{O}^{\nu}(\mathbf{p}, \mathbf{r})$ to $M P^{\nu}$, for all $t$ and all $\nu \in \mathcal{N}$, it must be $\frac{v_{i}^{\prime}\left(c_{t}^{* \nu}\right)}{v_{j}^{\prime}\left(c_{t}^{* \nu}\right)}=\frac{p_{i t}}{p_{j t}}$ and $\phi^{\prime}\left(L-\Lambda_{t}^{* \nu}\right)=w_{t}^{\max } \frac{v_{i}^{\prime}\left(c_{t}^{* \nu}\right)}{p_{i t}}$ for all $i, j$. By the linear homogeneity of $v($.$) , this implies that at$ an interior solution to $M P^{\nu}$, at all $t$, it must be $\frac{c_{i t}^{* \nu}}{c_{j t}^{* \nu}}=\frac{c_{i t}^{* \mu}}{c_{j t}^{* \mu}}$ for all $\nu, \mu \in \mathcal{N}$, and therefore $v_{i}^{\prime}\left(c_{t}^{* \nu}\right)=v_{i}^{\prime}\left(c_{t}^{* \mu}\right)$ and $\phi^{\prime}\left(L-\Lambda_{t}^{* \nu}\right)=\phi^{\prime}\left(L-\Lambda_{t}^{* \mu}\right)$ for all $\nu, \mu \in \mathcal{N}$. The first part of the statement then follows from the strict concavity of $\phi$.

2. At any $t$, let $c_{t}^{*} \in \mathbb{R}_{+}^{n}$ be such that $\frac{v_{i}^{\prime}\left(c_{t}^{*}\right)}{v_{j}^{\prime}\left(c_{t}^{*}\right)}=\frac{p_{i t}}{p_{j t}}$, for all $i, j$. Then by step 1 , at an IRS, it must be $c_{t}^{* \nu}=k_{t}^{\nu} c_{t}^{*}$ where $k_{t}^{\nu}=\frac{\left(1+r_{t}\right) p_{t-1} \omega_{t}^{\nu}+w_{t}^{\max } \Lambda_{t}^{*}-p_{t} \omega_{t+1}^{\nu}}{p_{t} c_{t}^{*}}>0$, at all $t$ and for all $\nu \in \mathcal{N}$. Take any two adjacent periods $t, t+1$, and consider $\nu \in \mathcal{N}$ such that $p_{t} \omega_{t+1}^{\nu}>0$. Consider a small one-period perturbation of $\omega^{\nu}$ such that $\mathrm{d} k_{t}^{\nu}=-\frac{1}{p_{t} c_{t}^{*}} p_{t} \mathrm{~d} \omega_{t+1}^{\nu}$ and $\mathrm{d} k_{t+1}^{\nu}=\frac{\left(1+r_{t+1}\right)}{p_{t+1} c_{t+1}^{*}} p_{t} \mathrm{~d} \omega_{t+1}^{\nu}$. By the linear homogeneity of $v$, the resulting change in welfare is $v\left(c_{t}^{*}\right) \mathrm{d} k_{t}^{\nu}+\rho v\left(c_{t+1}^{*}\right) \mathrm{d} k_{t+1}^{\nu}=-v\left(c_{t}^{*}\right) \frac{1}{p_{t} c_{t}^{*}} p_{t} \mathrm{~d} \omega_{t+1}^{\nu}+v\left(c_{t+1}^{*}\right) \rho \frac{\left(1+r_{t+1}\right)}{p_{t+1} c_{t+1}^{*}} p_{t} \mathrm{~d} \omega_{t+1}^{\nu}$.

3. By step 1 , at an IRS, at all $t, \phi^{\prime}\left(L-\Lambda_{t}^{*}\right)=w_{t}^{\max } \frac{v_{i}^{\prime}\left(c_{\nu}^{* \nu}\right)}{p_{i t}}$ for all $i$ and all $\nu \in \mathcal{N}$. By the linear homogeneity of $v$, this implies that $\phi^{\prime}\left(L-\Lambda_{t}^{*}\right)=w_{t}^{\max } \frac{v\left(c_{t}^{* \nu}\right)}{p_{t} c_{t}^{* \nu}}=w_{t}^{\max } \frac{v\left(c_{t}^{*}\right)}{p_{t} c_{t}^{*}}$. Using the latter expression, the change in welfare can be written equivalently as $-\frac{\phi^{\prime}\left(L-\Lambda_{t}^{*}\right)}{w_{t}^{\max }} p_{t} \mathrm{~d} \omega_{t+1}^{\nu}+$ $\rho\left(1+r_{t+1}\right) \frac{\phi^{\prime}\left(L-\Lambda_{t+1}^{*}\right)}{w_{t+1}^{\max }} p_{t} \mathrm{~d} \omega_{t+1}^{\nu}$. Therefore a necessary condition for $\xi^{\nu} \in \mathcal{O}^{\nu}(\mathbf{p}, \mathbf{r})$ to be an interior solution to $M P^{\nu}$ is that $\left[-\frac{\phi^{\prime}\left(L-\Lambda_{t}^{*}\right)}{w_{t}^{\max }}+\rho\left(1+r_{t+1}\right) \frac{\phi^{\prime}\left(L-\Lambda_{t+1}^{*}\right)}{w_{t+1}^{\max }}\right] p_{t} \mathrm{~d} \omega_{t+1}^{\nu} \leqq 0$ for all $\mathrm{d} \omega_{t+1}^{\nu}$, which holds only if the expression in brackets is equal to zero.

\section{The International Class Structure}

In this section, we derive the international class structure, where "classes of countries can be defined with reference to the use of the credit market" (Roemer [22], p.54). Let $\left(a_{1}, a_{2}, a_{3}\right)$ be a vector where $a_{i} \in\{+, \mathbf{0}\}, i=1,3, a_{2} \in\{+, 0\}$, and "+" means a non-zero vector in the appropriate place. We extend Roemer's definition of classes within each period $t$.

Definition 3: Let $\left((\mathbf{p}, \mathbf{r}),\left(\xi^{\nu}\right)_{\nu \in \mathcal{N}}\right)$ be a RS for $E\left(\Omega_{0}\right)$. Country $\nu$ is said to be a member of class $\left(a_{1}, a_{2}, a_{3}\right)$ within period $t$, or $W P_{t}$ class $\left(a_{1}, a_{2}, a_{3}\right)$, if there is a $\xi^{\prime \nu} \in \mathcal{O}^{\nu}(\mathbf{p}, \mathbf{r})$ with $\omega^{\prime \nu}=\omega^{\nu}$ such that $\left(\alpha_{t}^{\prime \nu}, z_{t}^{\prime \nu}, \beta_{t}^{\prime \nu}\right)$ has the form $\left(a_{1}, a_{2}, a_{3}\right)$ in $t$.

There are eight conceivable classes $\left(a_{1}, a_{2}, a_{3}\right)$, but only four of them are theoretically 
relevant, as argued by Roemer [21]. At a $\operatorname{RS}\left((\mathbf{p}, \mathbf{r}),\left(\xi^{\nu}\right)_{\nu \in \mathcal{N}}\right)$ for $E\left(\Omega_{0}\right)$, at all $t$, let $\Gamma_{t}^{\nu}=$ $\left\{\left(\alpha_{t}^{\prime \nu}, z_{t}^{\prime \nu}, \beta_{t}^{\prime \nu}\right) \mid \xi^{\prime \nu} \in \mathcal{O}^{\nu}(\mathbf{p}, \mathbf{r})\right.$ with $\left.\omega^{\prime \nu}=\omega^{\nu}\right\}$. We say that $\Gamma_{t}^{\nu}$ has a solution of the form $\left(a_{1}, a_{2}, a_{3}\right) \backslash\left(a_{1}^{\prime}, a_{2}^{\prime}, a_{3}^{\prime}\right)$ to mean that $\Gamma_{t}^{\nu}$ contains a vector $\left(\alpha_{t}^{\prime \nu}, z_{t}^{\prime \nu}, \beta_{t}^{\prime \nu}\right)$ of the form $\left(a_{1}, a_{2}, a_{3}\right)$ but not one of the form $\left(a_{1}^{\prime}, a_{2}^{\prime}, a_{3}^{\prime}\right)$. Then:

$$
\begin{aligned}
& C_{t}^{1}=\left\{\nu \in \mathcal{N} \mid \Gamma_{t}^{\nu} \text { has a solution of the form }(+,+, \mathbf{0}) \backslash(+, 0, \mathbf{0})\right\}, \\
& C_{t}^{2}=\left\{\nu \in \mathcal{N} \mid \Gamma_{t}^{\nu} \text { has a solution of the form }(+, 0, \mathbf{0})\right\}, \\
& C_{t}^{3}=\left\{\nu \in \mathcal{N} \mid \Gamma_{t}^{\nu} \text { has a solution of the form }(+, 0,+) \backslash(+, 0, \mathbf{0})\right\}, \\
& C_{t}^{4}=\left\{\nu \in \mathcal{N} \mid \Gamma_{t}^{\nu} \text { has a solution of the form }(\mathbf{0}, 0,+)\right\} .
\end{aligned}
$$

At all $t$, countries in $C_{t}^{1}$ are net lenders in the global market; countries in $C_{t}^{2}$ can optimise without using the capital market; countries in $C_{t}^{3}$ must borrow foreign capital to optimise; countries in $C_{t}^{4}$ must borrow all of their operating capital. This definition of classes based on credit relations conveys the intuition that a country's position in the capital market affects its international status.

Below, it is proved that in equilibrium the set of countries $\mathcal{N}$ can indeed be partitioned into these four $W P_{t}$ classes at all $t$. First, Lemma 3 proves that $W P_{t}$ classes $(+,+,+)$ and $(\mathbf{0},+,+)$ can be ignored.

Lemma 3: Let $\left((\mathbf{p}, \mathbf{r}),\left(\xi^{\nu}\right)_{\nu \in \mathcal{N}}\right)$ be an IRS for $E\left(\Omega_{0}\right)$. Let $\xi^{\nu} \in \mathcal{O}^{\nu}(\mathbf{p}, \mathbf{r})$ be such that $\nu$ is a member of $W P_{t}$ class $(+,+,+)$ or $(\mathbf{0},+,+)$ in $t$. Then:

$$
\begin{aligned}
& \text { if } z_{t}^{\prime \nu}>p_{t-1} \underline{\beta}_{t}^{\prime \nu} \text { for all }\left(\alpha_{t}^{\prime \nu}, z_{t}^{\prime \nu}, \beta_{t}^{\prime \nu}\right) \in \Gamma_{t}^{\nu} \text {, then } \nu \in C_{t}^{1} \text {; } \\
& \text { if } z_{t}^{\prime \nu}=p_{t-1} \underline{\beta}_{t}^{\prime \nu} \text { for some }\left(\alpha_{t}^{\prime \nu}, z_{t}^{\prime \nu}, \beta_{t}^{\prime \nu}\right) \in \Gamma_{t}^{\nu} \text {, then } \nu \in C_{t}^{2} \text {; } \\
& \text { if } z_{t}^{\prime \nu}<p_{t-1} \underline{\beta}_{t}^{\prime \nu} \text { for all }\left(\alpha_{t}^{\prime \nu}, z_{t}^{\prime \nu}, \beta_{t}^{\prime \nu}\right) \in \Gamma_{t}^{\nu} \text {, then } \nu \in C_{t}^{3} \text {. }
\end{aligned}
$$

Proof: 1 . By the convexity of $M P^{\nu}$, it follows that if $z_{t}^{\prime \nu}<p_{t-1} \underline{\beta}_{t}^{\prime \nu}$ for some $\left(\alpha_{t}^{\prime \nu}, z_{t}^{\prime \nu}, \beta_{t}^{\prime \nu}\right) \in$ $\Gamma_{t}^{\nu}$ and $z_{t}^{\prime \prime \nu}>p_{t-1} \underline{\beta}_{t}^{\prime \prime \nu}$ for some other $\left(\alpha_{t}^{\prime \prime \nu}, z_{t}^{\prime \prime \nu}, \beta_{t}^{\prime \prime \nu}\right) \in \Gamma_{t}^{\nu}$, then there exists $\left(\alpha_{t}^{\prime \prime \prime \nu}, z_{t}^{\prime \prime \prime \nu}, \beta_{t}^{\prime \prime \prime \nu}\right) \in$ $\Gamma_{t}^{\nu}$ such that $z_{t}^{\prime \prime \prime}=p_{t-1} \underline{\beta}_{t}^{\prime \prime \prime}$. Therefore, the three cases in the statement are mutually 
exclusive and they decompose the set of agents with $W_{t}^{\nu}>0$ into disjoint sets.

2. Suppose $z_{t}^{\prime \nu}>p_{t-1} \underline{\beta}_{t}^{\prime \nu}$ for all $\left(\alpha_{t}^{\prime \nu}, z_{t}^{\prime \nu}, \beta_{t}^{\prime \nu}\right) \in \Gamma_{t}^{\nu}$. Construct $\left(\alpha_{t}^{\prime \nu}, z_{t}^{\prime \nu}, \beta_{t}^{\prime \nu}\right)$ such that $\alpha_{t}^{\prime \nu}=\alpha_{t}^{\nu}+\beta_{t}^{\nu}, z_{t}^{\prime \nu}=z_{t}^{\nu}-p_{t-1} \underline{\beta}_{t}^{\nu}>0$, and $\beta_{t}^{\prime \nu}=\mathbf{0}$. By the convexity of $P, \alpha_{t}^{\prime \nu} \in P$, $\alpha_{l t}^{\prime \nu}=\alpha_{l t}^{\nu}+\beta_{l t}^{\nu}$ and $z_{t}^{\prime \nu}+p_{t-1} \underline{\alpha}_{t}^{\nu \nu}=z_{t}^{\nu}+p_{t-1} \underline{\alpha}_{t}^{\nu}$. Then by Lemma $1,\left(\alpha_{t}^{\prime \nu}, z_{t}^{\prime \nu}, \beta_{t}^{\prime \nu}\right) \in \Gamma_{t}^{\nu}$. Thus, it remains to show that there is no solution in $\Gamma_{t}^{\nu}$ of the form $(+, 0, \mathbf{0})$. Suppose, by way of contradiction, that $\Gamma_{t}^{\nu}$ contains a solution of the form $(+, 0,0)$ at $t$. Construct $\left(\alpha_{t}^{\prime \nu}, z_{t}^{\prime \nu}, \beta_{t}^{\prime \nu}\right)$ such that $\alpha_{t}^{\prime \nu}=\mathbf{0}, z_{t}^{\prime \nu}=p_{t-1} \underline{\alpha}_{t}^{\nu}>0$, and $\beta_{t}^{\prime \nu}=\alpha_{t}^{\nu}+\beta_{t}^{\nu}$. Clearly, $\beta_{t}^{\prime \nu} \in P, \beta_{l t}^{\prime \nu}=\alpha_{l t}^{\nu}+\beta_{l t}^{\nu}$ and $z_{t}^{\prime \nu}+p_{t-1} \underline{\alpha}_{t}^{\prime \nu}=z_{t}^{\nu}+p_{t-1} \underline{\alpha}_{t}^{\nu}$. Then by Lemma $1,\left(\alpha_{t}^{\prime \nu}, z_{t}^{\prime \nu}, \beta_{t}^{\prime \nu}\right) \in \Gamma_{t}^{\nu}$, contradicting the assumption that $z_{t}^{\prime \nu}>p_{t-1} \underline{\beta}_{t}^{\prime \nu}$ for all $\left(\alpha_{t}^{\prime \nu}, z_{t}^{\prime \nu}, \beta_{t}^{\prime \nu}\right) \in \Gamma_{t}^{\nu}$.

3. The other two cases are proved similarly.

Lemma 3 substantiates the claim that a country's class status is determined by its position in the capital market: net lenders form the 'upper' strata of the global economy, whereas net borrowers occupy 'lower' positions. It is therefore natural to investigate whether class status (and so a country's position in the credit market) is determined by its wealth.

Consider an IRS. At all $t$, let $\alpha_{t}^{\min }$ be defined as follows: $\alpha_{t}^{\min } \in P_{t}^{w}(\mathbf{p}, \mathbf{r}), \frac{p_{t-1} \underline{\alpha}_{t}^{\min }}{\alpha_{l t}^{\min }}=$ $\min _{\alpha \in P_{t}^{w}(\mathbf{p}, \mathbf{r})}\left[\frac{p_{t-1} \underline{\alpha}}{\alpha_{l}}\right]$, and $\alpha_{l t}^{\min }=\Lambda_{t}^{*}$, where $\Lambda_{t}^{*}>0$ is defined in Lemma 2. Similarly, $\alpha_{t}^{\max }$ is given by: $\alpha_{t}^{\max } \in P_{t}^{w}(\mathbf{p}, \mathbf{r}), \frac{p_{t-1} \underline{\alpha}_{t}^{\max }}{\alpha_{l t}^{\max }}=\max _{\alpha \in P_{t}^{w}(\mathbf{p}, \mathbf{r})}\left[\frac{p_{t-1} \underline{\alpha}}{\alpha_{l}}\right]$, and $\alpha_{l t}^{\max }=\Lambda_{t}^{*}$. Note that $p_{t-1} \underline{\alpha}_{t}^{\min } \leqq p_{t-1} \underline{\alpha}_{t}^{\max }$ and that $\alpha_{t}^{\min }, \alpha_{t}^{\max }$ are well-defined. Theorem 1 generalises one of the main results of Roemer's theory of classes: at an IRS, $W P_{t}$ classes are pairwise disjoint and exhaustive, and $W P_{t}$ class status depends on a country's wealth. ${ }^{14}$

Theorem 1 (The Dependence School Theorem): Let $\left((\mathbf{p}, \mathbf{r}),\left(\xi^{\nu}\right)_{\nu \in \mathcal{N}}\right)$ be an IRS for $E\left(\Omega_{0}\right)$ such that $1+r_{t}>\max _{i} \frac{p_{i t}}{p_{i t-1}}$ for all $t$. Then at all $t$ :

(i) $\nu \in C_{t}^{1} \Leftrightarrow p_{t-1} \underline{\alpha}_{t}^{\max }<W_{t}^{\nu}$;

(ii) $\nu \in C_{t}^{2} \Leftrightarrow p_{t-1} \underline{\alpha}_{t}^{\min } \leqq W_{t}^{\nu} \leqq p_{t-1} \underline{\alpha}_{t}^{\max }$

(iii) $\nu \in C_{t}^{3} \Leftrightarrow 0<W_{t}^{\nu}<p_{t-1} \underline{\alpha}_{t}^{\min }$;

(iv) $\nu \in C_{t}^{4} \Leftrightarrow W_{t}^{\nu}=0$.

\footnotetext{
${ }^{14}$ The condition $1+r_{t}>\max _{i} \frac{p_{i t}}{p_{i t-1}}$ ensures that undertaking production activities is better than storing goods to be sold at the end of the period. In order to interpret this condition, note that at a stationary IRS with $p_{t}=p_{t-1}$ it reduces to the familiar requirement that $r_{t}>0$.
} 
Proof: 1. By Lemma 2, at an IRS, $\Lambda_{t}^{\nu}=\Lambda_{t}^{*}=\alpha_{l t}^{\nu}+\beta_{l t}^{\nu}>0$, all $\nu \in \mathcal{N}$.

2. By step 1, it immediately follows that at any $t, \nu \in C_{t}^{4}$ if and only if $W_{t}^{\nu}=0$.

3. Consider part (ii). Suppose $p_{t-1} \underline{\alpha}_{t}^{\min } \leqq W_{t}^{\nu} \leqq p_{t-1} \underline{\alpha}_{t}^{\max }$. We show that $\Gamma_{t}^{\nu}$ has a solution of the form $(+, 0,0)$. By step 1 , and noting that $1+r_{t}>0$, at an IRS it must be $w_{t}^{\max } \Lambda_{t}^{*}+\left(1+r_{t}\right) W_{t}^{\nu}=p_{t} c_{t}^{* \nu}+p_{t} \omega_{t+1}^{\nu}$, and any $\left(\alpha_{t}^{\prime \nu}, z_{t}^{\prime \nu}, \beta_{t}^{\prime \nu}\right)$ with $\alpha_{t}^{\prime \nu}, \beta_{t}^{\prime \nu} \in P_{t}^{w}(\mathbf{p}, \mathbf{r})$, $\alpha_{l t}^{\prime \nu}+\beta_{l t}^{\prime \nu}=\Lambda_{t}^{*}, z_{t}^{\prime \nu} \geqq 0$, and $p_{t-1} \underline{\alpha}_{t}^{\prime \nu}+z_{t}^{\prime \nu}=W_{t}^{\nu}$ is part of an optimal solution. But then, since $p_{t-1} \underline{\alpha}_{t}^{\min } \leqq W_{t}^{\nu} \leqq p_{t-1} \underline{\alpha}_{t}^{\max }$, by the convexity of $P$, it follows that there exists some $\alpha_{t}^{\prime \prime \nu} \in P_{t}^{w}(\mathbf{p}, \mathbf{r})$, such that $w_{t}^{\max } \alpha_{l t}^{\prime \prime \nu}+\left(1+r_{t}\right) p_{t-1} \underline{\alpha}_{t}^{\prime \prime \nu}=p_{t} c_{t}^{* \nu}+p_{t} \omega_{t+1}^{\nu}$, with $\alpha_{l t}^{\prime \prime \nu}=\Lambda_{t}^{*}$ and $p_{t-1} \underline{\alpha}_{t}^{\prime \prime \nu}=W_{t}^{\nu}$. Conversely, if $\nu \in C_{t}^{2}$, then there exists $\alpha_{t} \in P_{t}^{w}(\mathbf{p}, \mathbf{r})$ such that $\left(1+r_{t}\right) p_{t-1} \underline{\alpha}_{t}+w_{t}^{\max } \alpha_{l t}=p_{t} c_{t}^{* \nu}+p_{t} \omega_{t+1}^{\nu}$, with $p_{t-1} \underline{\alpha}_{t}=W_{t}^{\nu}$ and $\alpha_{l t}=\Lambda_{t}^{*}$, which implies $p_{t-1} \underline{\alpha}_{t}^{\min } \leqq W_{t}^{\nu} \leqq p_{t-1} \underline{\alpha}_{t}^{\max }$.

4. Parts (i) and (iii) are proved similarly.

In order to clarify the normative relevance of Theorem 1 , note that country $\nu$ 's wealth, $W_{t}^{\nu}$, can be seen as the main proxy for its level of development. Theorem 1 implies that in equilibrium less developed countries (with a lower $W_{t}^{\nu}$ ) are net borrowers, whereas developed countries (with a higher $W_{t}^{\nu}$ ) are net lenders: a nation's wealth (and development) level determines its class status. Given that international markets for commodities and capital are perfectly competitive, in equilibrium all countries enjoy benefits from trade, as international capital flows allow poor countries to improve their lot. Yet, the IRS is also characterised by a four-class structure which reflects the wealth hierarchy and an asymmetric relationship between countries. For the economic development of the countries in $C_{t}^{3} \cup C_{t}^{4}$ is crucially dependent on the existence of the rich countries in $C_{t}^{1}$ which export their capital to the poor, whereas the rich in $C_{t}^{1}$ could realise a certain economic development with full employment by themselves alone. In this sense, Theorem 1 captures some of the key insights of the 'dependence school' discussed in the Introduction, and is named accordingly.

As noted by an anonymous referee, Theorem 1 echoes a standard result in neoclassical models in which foreign capital flows into underdeveloped countries because their endowment of capital per capita is lower and therefore its marginal product is higher. At a closer look, 
however, some important differences emerge. Although our assumptions on technology allow for smooth production sets, we are not postulating the existence of a differentiable aggregate production function and therefore cannot derive any conclusions concerning the marginal productivity of capital. The world interest rate is unique because of perfect competition in the capital market but in principle there may be multiple equilibrium values of $r_{t}$ in any period, none of which necessarily linked to a notion of marginal product of capital. Capital flows are not driven by the search of the investment yielding the highest return: they are determined by agents' optimising choices on consumption, savings and leisure. Thus, for example, in any $t$, a wealthy nation uses its capital to finance internal production up to the point where its citizens are spending the optimal amount of labour. It then lends any remaining capital - at the given international rate - in order to increase consumption, or savings. (And a similar logic drives borrowing decisions in poor countries.)

\section{Exploitative International Relations}

Exploitation in international relations is conceived of as the unequal exchange of labour between countries as in Roemer [21, 22]: exploitative international relations are characterised by systematic differences between the labour 'contributed' by agents in country $\nu$ and the labour 'received' by them via their national income. As intuitive as this definition may seem, in general economies the notions of labour 'contributed' and labour 'received' are not obvious. Indeed, the very existence of a general, consistent definition which preserves the key insights of UE theory has been put into doubt. In this section, we develop an axiomatic analysis of UE exploitation and characterise a class of definitions that satisfy three important properties. In the next section, we prove that the class is nonempty.

\subsection{A domain axiom}

In economies with homogeneous labour, the labour 'contributed' by $\nu$ coincides with the labour performed by workers in $\nu, \Lambda_{t}^{\nu} \cdot{ }^{15}$ Outside of static, two-class Leontief economies

\footnotetext{
${ }^{15}$ For a generalisation to economies with heterogeneous labour, see Veneziani and Yoshihara [30, 29].
} 
with subsistence wages, instead, many different definitions of the labour 'received' by $\nu$ can be, and have in fact been proposed, which incorporate different normative and positive views. ${ }^{16}$ In recent work, Yoshihara and Veneziani [33, 31, 30, 29] have proposed an axiom that identifies the domain of admissible UE definitions: it imposes some weak restrictions on the notion of labour received and all of the main approaches satisfy it in static economies. In this subsection, we generalise it to the dynamic context.

At the most general level, in UE theory, the amount of labour 'received' by agent $\nu$ is determined with reference to some bundles that belong to a normatively relevant budget set and capture $\nu$ 's consumption opportunities. In static models, all of the main UE approaches usually (albeit sometimes implicitly) assume that in equilibrium agents at least replace any wealth used up in production in every period and stipulate that the reference bundles are, or can be purchased with agents' actual net incomes at all $t$. In the general dynamic framework considered here, the relevant notion of agents' net income is not obvious and the choice of the appropriate budget set is not unambiguous.

Let $\left((\mathbf{p}, \mathbf{r}),\left(\xi^{\nu}\right)_{\nu \in \mathcal{N}}\right)$ be a RS for $E\left(\Omega_{0}\right)$. The gross income of each $\nu \in \mathcal{N}$ at $t$ is given by $\left(1+r_{t}\right) p_{t-1} \omega_{t}^{\nu}+w_{t}^{\max } \Lambda_{t}^{\nu}$. In order to identify $\nu$ 's 'net' income at $t$ in this context, the fund for replenishing $\nu$ 's wealth $p_{t-1} \omega_{t}^{\nu}$ in the next period should be deducted after adjusting for the difference in prices between $t-1$ and $t$. To do so, we define the inflation index at $t, R_{t} \equiv \frac{p_{t} \omega_{t}}{p_{t-1} \omega_{t}}$, taking $\omega_{t}$ as the inflation basket. Given this index, $\nu$ 's wealth $p_{t-1} \omega_{t}^{\nu}$ at $t-1$ is evaluated as being equivalent to $R_{t} p_{t-1} \omega_{t}^{\nu}$ at $t$. Then, $\nu$ 's 'net' income at $t$ can be defined as $\left(1+r_{t}\right) p_{t-1} \omega_{t}^{\nu}+w_{t}^{\max } \Lambda_{t}^{\nu}-R_{t} p_{t-1} \omega_{t}^{\nu}$, and it identifies the normatively relevant set of commodity bundles 'received' by $\nu$ :

$$
B_{t}\left((\mathbf{p}, \mathbf{r}) ; p_{t-1} \omega_{t}^{\nu}, \Lambda_{t}^{\nu}\right) \equiv\left\{c \in \mathbb{R}_{+}^{n} \mid p_{t} c=\left(1+r_{t}\right) p_{t-1} \omega_{t}^{\nu}+w_{t}^{\max } \Lambda_{t}^{\nu}-R_{t} p_{t-1} \omega_{t}^{\nu}\right\}
$$

In other words, in a general dynamic context the set of commodity bundles 'received' by $\nu$ at $t$ is defined counterfactually by considering the net income that could be devoted to consumption if $\nu$ decided only to replace its wealth, i.e. to carry forward the real asset value of

\footnotetext{
${ }^{16}$ See, for example, Morishima [17] and Roemer [21]. See Yoshihara [31, 32] and Veneziani and Yoshihara [29] for a thorough discussion.
} 
$W_{t}^{\nu}$ to the next period. ${ }^{17}$ The reason for this choice is threefold. First, countries are interested in wealth, rather than in a specific vector of capital endowments. Second, from a normative perspective, for a given gross income, in every $t$ exploitation status should not depend on specific saving and investment decisions, or on the specific vector of productive endowments purchased. According to Roemer ([22], p.53), for example, the appropriate notion of UE exploitation should be preference-independent. Third, it is immediate to show that the focus on bundles in $B_{t}\left((\mathbf{p}, \mathbf{r}) ; p_{t-1} \omega_{t}^{\nu}, \Lambda_{t}^{\nu}\right)$ is a generalisation of the standard approach and it reduces to the latter at a RS with stationary prices and capital.

Let $\mathcal{E}$ denote the set of all economies $E\left(P, \mathcal{N}, u, \rho, \Omega_{0}\right)$ that satisfy our assumptions on technology, agents, preferences and endowments. For all $c \in \mathbb{R}_{+}^{n}$, let $\psi(c) \equiv\{\alpha \in P \mid \bar{\alpha} \geq c\}$ be the set of production activities that can produce $c$ as a portion of output. Given any definition of exploitation, let $\mathcal{N}_{t}^{\text {ter }} \subseteq \mathcal{N}$ and $\mathcal{N}_{t}^{\text {ted }} \subseteq \mathcal{N}$ denote, respectively, the set of exploiters at $t$, or $W P_{t}$ exploiters, and the set of exploited agents at $t$, or $W P_{t}$ exploited agents, at a given allocation. Our domain axiom can now be formally introduced.

Labour Exploitation (LE): Consider any economy $E\left(\Omega_{0}\right) \in \mathcal{E} . \quad$ Let $\left((\mathbf{p}, \mathbf{r}),\left(\xi^{\nu}\right)_{\nu \in \mathcal{N}}\right)$ be a RS for $E\left(\Omega_{0}\right)$. Given any definition of UE exploitation, in each period $t$ two subsets $\mathcal{N}_{t}^{\text {ter }} \subseteq \mathcal{N}$ and $\mathcal{N}_{t}^{\text {ted }} \subseteq \mathcal{N}, \mathcal{N}_{t}^{\text {ter }} \cap \mathcal{N}_{t}^{\text {ted }}=\varnothing$, constitute the set of $W P_{t}$ exploiters and the set of $W P_{t}$ exploited agents if and only if for any $\nu \in \mathcal{N}$, there exist $\bar{c}_{t}^{\nu}, \underline{c}_{t}^{\nu} \in B_{t}\left((\mathbf{p}, \mathbf{r}) ; p_{t-1} \omega_{t}^{\nu}, \Lambda_{t}^{\nu}\right)$ such that there exist $\alpha^{\bar{c}_{t}^{\nu}} \in \psi\left(\bar{c}_{t}^{\nu}\right) \cap \partial P$ with $p_{t} \widehat{\alpha}^{\bar{c}_{t}^{\nu}} \geqq p_{t} \bar{c}_{t}^{\nu}$ and $\alpha^{\underline{c}_{t}^{\nu}} \in \psi\left(\underline{c}_{t}^{\nu}\right) \cap \partial P$ with $p_{t} \widehat{\alpha}^{\underline{c}_{t}^{\nu}} \geqq p_{t} \underline{c}_{t}^{\nu}$ such that $\alpha_{l}^{c_{t}^{\nu}} \geqq \alpha_{l}^{\bar{c}_{t}^{\nu}}$, and the following condition holds,

$$
\begin{aligned}
& \nu \in \mathcal{N}_{t}^{t e r} \Leftrightarrow \Lambda_{t}^{\nu}<\alpha_{l}^{\bar{c}_{t}^{\nu}} ; \\
& \nu \in \mathcal{N}_{t}^{t e d} \Leftrightarrow \Lambda_{t}^{\nu}>\alpha_{l}^{c_{t}^{\nu}} .
\end{aligned}
$$

LE requires UE exploitation status to be determined based on the labour contributed by countries (the labour performed by their citizens) and on the labour received by them, where the latter is determined in relation both to purchasing power, and to productive conditions.

\footnotetext{
${ }^{17}$ The set $B_{t}\left((\mathbf{p}, \mathbf{r}) ; p_{t-1} \omega_{t}^{\nu}, \Lambda_{t}^{\nu}\right)$ does not necessarily contain $\nu$ 's actual consumption bundle at $t$, as $p_{t} \omega_{t+1}^{\nu}$ may be different from $R_{t} p_{t-1} \omega_{t}^{\nu}$, in equilibrium.
} 
To be specific, by LE under any admissible definition, in equilibrium the sets $\mathcal{N}_{t}^{t e r}$, $\mathcal{N}_{t}^{\text {ted }}$ are characterised in each $t$ by identifying two (possibly identical) reference bundles $\bar{c}_{t}^{\nu}, \underline{c}_{t}^{\nu} \in \mathbb{R}_{+}^{n}$ for each $\nu \in \mathcal{N}$. The reference bundles must be affordable for any $\nu$ who simply replaces its wealth at $t\left(\bar{c}_{t}^{\nu}, \underline{c}_{t}^{\nu} \in B_{t}\left((\mathbf{p}, \mathbf{r}) ; p_{t-1} \omega_{t}^{\nu}, \Lambda_{t}^{\nu}\right)\right)$ and must be technically producible as a portion of the output of efficient production activities while carrying forward the real asset value of the commodity inputs of such production activities to the next period $\left(\alpha^{c} \in \psi(c) \cap \partial P\right.$ with $\left.p_{t} \widehat{\alpha}^{c} \geqq p_{t} c, c=\bar{c}_{t}^{\nu}, \underline{c}_{t}^{\nu}\right){ }^{18}$ The labour contained in $\bar{c}_{t}^{\nu}, \underline{c}_{t}^{\nu}$ is equal to the amount of labour required to produce them as a portion of output: respectively, $\alpha_{l}^{\bar{c}_{t}^{\nu}}, \alpha_{l}^{\frac{c_{t}^{\nu}}{t}}$. Given $\alpha_{l}^{c_{t}^{\nu}} \geqq \alpha_{l}^{\bar{c}_{t}^{\nu}}$, the (possibly degenerate) interval $\left[\alpha_{l}^{\bar{c}_{t}^{\nu}}, \alpha_{l}^{c_{t}^{\nu}}\right]$ is the labour that $\nu$ can receive via its 'net income' at $t$ and it determines $\nu$ 's UE exploitation status at $t$, once compared with the labour contributed by $\nu, \Lambda_{t}^{\nu}$. In equilibrium, at any $t, \nu$ is a $W P_{t}$ exploiter if and only if $\nu$ works less than the minimum amount of labour that $\nu$ can receive via its 'net income', $\alpha_{l}^{\bar{c}_{t}^{\nu}}$; whereas $\nu$ is $W P_{t}$ exploited if and only if $\nu$ works more than the maximum amount of labour that $\nu$ can receive via its 'net income', $\alpha_{l}^{\frac{c_{t}^{\nu}}{t}}$.

LE is a weak condition that captures some fundamental insights of UE theory shared by all of the main approaches in the literature. ${ }^{19}$ It identifies the domain of admissible UE definitions, but it cannot discriminate among alternative definitions within this domain, which can be large indeed. For this purpose, some additional properties must be imposed.

\subsection{Class, Wealth and Exploitation}

A fundamental insight of UE theory is the existence of a relation between development or wealth, - exploitation status, and class position in the global economy. The existence of such a relation is often proved as a result in a given economic environment, under certain conditions. Yet its central relevance in UE theory is such that "its epistemological status in our understanding is as a postulate. We seek a model which will make our postulated

\footnotetext{
${ }^{18}$ Note that $p_{t} \widehat{\alpha}^{c} \geqq p_{t} c$ implies $p_{t} \underline{\alpha}^{c} \leqq p_{t} \bar{\alpha}^{c}-p_{t} c$, where the left hand side represents the real asset value of the commodity inputs of production activity $\alpha^{c}$.

${ }^{19}$ In particular, it is worth noting that axiom LE does not require UE exploitation status to be defined based on imputing embodied labor magnitudes to exchanged commodity bundles as in standard approaches. But nor does it rule out the possibility that the labour received by $\nu$ corresponds to the labour embodied in a specific bundle. We are grateful to an anonymous referee for pointing this out.
} 
belief true" (Roemer [21], p.152). In this subsection, we state this intuition axiomatically and formalise two properties that incorporate the relation between wealth, class, and exploitation status. Then, we provide a characterisation of the class of definitions of UE exploitation that satisfy $\mathbf{L E}$ and both properties, in the dynamic international economies considered here.

The first property captures the intuition that richer countries are UE exploiters while less developed countries suffer from UE exploitation:

Wealth-Exploitation Correspondence Principle (WECP): Let $\left((\mathbf{p}, \mathbf{r}),\left(\xi^{\nu}\right)_{\nu \in \mathcal{N}}\right)$ be an IRS for $E\left(P, \mathcal{N}, u, \rho, \Omega_{0}\right)$ such that $1+r_{t}-R_{t}>0$ for all $t$. For each $t$, there exist $\bar{W}_{t}, \underline{W}_{t}>0$ with $\bar{W}_{t} \geqq \underline{W}_{t}$ such that for any $E\left(P, \mathcal{N}, u, \rho, \Omega_{0}^{\prime}\right) \in \mathcal{E}$ with $\sum_{\nu \in \mathcal{N}} \omega_{0}^{\prime \nu}=\sum_{\nu \in \mathcal{N}} \omega_{0}^{\nu}$ and any $\operatorname{IRS}\left((\mathbf{p}, \mathbf{r}),\left(\xi^{\prime \nu}\right)_{\nu \in \mathcal{N}}\right)$ for $E\left(P, \mathcal{N}, u, \rho, \Omega_{0}^{\prime}\right)$ with $\sum_{\nu \in \mathcal{N}} \omega_{t}^{\prime \nu}=\sum_{\nu \in \mathcal{N}} \omega_{t}^{\nu}$, all $t$ :

$$
\begin{aligned}
& W_{t}^{\prime \nu}>\bar{W}_{t} \Leftrightarrow \nu \in \mathcal{N}_{t}^{t e r} ; \\
& W_{t}^{\prime \nu}<\underline{W}_{t} \Leftrightarrow \nu \in \mathcal{N}_{t}^{t e d} .
\end{aligned}
$$

WECP states that, in equilibrium, in any given period there should be two (possibly equal) threshold wealth levels, $\bar{W}_{t}, \underline{W}_{t}$, such that the set of $W P_{t}$ exploiters (resp., $W P_{t}$ exploited) corresponds to the set of countries with wealth higher than $\bar{W}_{t}$ (resp., lower than $\left.\underline{W}_{t}\right)$. The threshold levels may depend on equilibrium prices and aggregate endowments, but not on the equilibrium wealth distribution.

The next Lemma characterises the set of definitions that satisfy WECP.

Lemma 4 (WECP): Let $\left((\mathbf{p}, \mathbf{r}),\left(\xi^{\nu}\right)_{\nu \in \mathcal{N}}\right)$ be an IRS for $E\left(P, \mathcal{N}, u, \rho, \Omega_{0}\right)$ such that $1+$ $r_{t}-R_{t}>0$ for all $t$. Given any definition of UE exploitation satisfying LE, the following statements are equivalent:

(i) WECP holds;

(ii) at all $t$, there exist $\bar{W}_{t}, \underline{W}_{t}>0$ with $\bar{W}_{t} \geqq \underline{W}_{t}$ such that for any $E\left(P, \mathcal{N}, u, \rho, \Omega_{0}^{\prime}\right) \in$ $\mathcal{E}$ with $\sum_{\nu \in \mathcal{N}} \omega_{0}^{\prime \nu}=\sum_{\nu \in \mathcal{N}} \omega_{0}^{\nu}$ and any $\operatorname{IRS}\left((\mathbf{p}, \mathbf{r}),\left(\xi^{\prime \nu}\right)_{\nu \in \mathcal{N}}\right)$ for $E\left(P, \mathcal{N}, u, \rho, \Omega_{0}^{\prime}\right)$ with 
$\sum_{\nu \in \mathcal{N}} \omega_{t}^{\prime \nu}=\sum_{\nu \in \mathcal{N}} \omega_{t}^{\nu}$, all $t$, and for each $\nu \in \mathcal{N}$

$$
\begin{aligned}
W_{t}^{\prime \nu}>\bar{W}_{t} & \Leftrightarrow W_{t}^{\prime \nu}>\frac{p_{t} \bar{c}_{t}^{\prime \nu}-w_{t}^{\max } \alpha_{l}^{\bar{c}_{t}^{\prime \nu}}}{1+r_{t}-R_{t}} ; \\
W_{t}^{\prime \nu}<\underline{W}_{t} & \Leftrightarrow W_{t}^{\prime \nu}<\frac{p_{t} \underline{c}_{t}^{\prime \nu}-w_{t}^{\max } \alpha_{l}^{\underline{c}_{t}^{\prime \nu}}}{1+r_{t}-R_{t}} .
\end{aligned}
$$

Proof: 1. Consider any economy $E\left(P, \mathcal{N}, u, \rho, \Omega_{0}^{\prime}\right) \in \mathcal{E}$ and any $\operatorname{IRS}\left((\mathbf{p}, \mathbf{r}),\left(\xi^{\prime \nu}\right)_{\nu \in \mathcal{N}}\right)$ for $E\left(P, \mathcal{N}, u, \rho, \Omega_{0}^{\prime}\right)$. Given a definition of exploitation satisfying $\mathbf{L E}$, at any $t$, for each $\nu \in \mathcal{N}$, there exist $\bar{c}_{t}^{\prime \nu}, \underline{c}_{t}^{\prime \nu} \in B_{t}\left((\mathbf{p}, \mathbf{r}) ; W_{t}^{\prime \nu}, \Lambda_{t}^{\prime \nu}\right)$ such that there exist $\alpha^{\bar{c}_{t}^{\prime \nu}} \in \psi\left(\bar{c}_{t}^{\prime \nu}\right) \cap \partial P$ with $p_{t} \widehat{\alpha}^{\bar{c}_{t}^{\prime \nu}} \geqq p_{t} \bar{c}_{t}^{\prime \nu}$ and $\alpha^{\underline{c}_{t}^{\prime \nu}} \in \psi\left(\underline{c}_{t}^{\prime \nu}\right) \cap \partial P$ with $p_{t} \widehat{\alpha}^{\underline{c}_{t}^{\prime \nu}} \geqq p_{t} \underline{\underline{c}}_{t}^{\prime \nu}$ such that $\alpha_{l}^{\underline{c}_{t}^{\prime \nu}} \geqq \alpha_{l}^{\bar{c}_{t}^{\prime \nu}}$ and $\nu \in \mathcal{N}_{t}^{t e r} \Leftrightarrow \Lambda_{t}^{\prime \nu}<\alpha_{l}^{\bar{c}_{t}^{\prime \prime}}$, and $\nu \in \mathcal{N}_{t}^{\text {ted }} \Leftrightarrow \Lambda_{t}^{\prime \nu}>\alpha_{l}^{c_{t}^{\prime \prime}}$.

2. In order to prove the result, it is sufficient to show that for any economy $E\left(P, \mathcal{N}, u, \rho, \Omega_{0}^{\prime}\right) \in$ $\mathcal{E}$ and any IRS $\left((\mathbf{p}, \mathbf{r}),\left(\xi^{\prime \nu}\right)_{\nu \in \mathcal{N}}\right)$ for $E\left(P, \mathcal{N}, u, \rho, \Omega_{0}^{\prime}\right)$, the following conditions hold at any $t$ and for each $\nu \in \mathcal{N}$,

$$
\begin{aligned}
& \nu \in \mathcal{N}_{t}^{t e r} \Leftrightarrow W_{t}^{\prime \nu}>\frac{p_{t} \bar{c}_{t}^{\prime \nu}-w_{t}^{\max } \alpha_{l}^{\bar{c}_{t}^{\prime \nu}}}{1+r_{t}-R_{t}} ; \\
& \nu \in \mathcal{N}_{t}^{t e d} \Leftrightarrow W_{t}^{\prime \nu}<\frac{p_{t} \underline{c}_{t}^{\prime \nu}-w_{t}^{\max } \alpha_{l}^{c_{t}^{\prime \prime}}}{1+r_{t}-R_{t}} .
\end{aligned}
$$

Consider (5). By LE, $\nu \in \mathcal{N}_{t}^{t e r} \Leftrightarrow \Lambda_{t}^{\prime \nu}<\alpha_{l}^{\bar{c}_{t}^{\prime \nu}}$. Moreover, $\bar{c}_{t}^{\prime \nu} \in B_{t}\left((\mathbf{p}, \mathbf{r}) ; W_{t}^{\prime \nu}, \Lambda_{t}^{\prime \nu}\right)$ implies $p_{t} \bar{c}_{t}^{\prime \nu}=\left(1+r_{t}-R_{t}\right) W_{t}^{\prime \nu}+w_{t}^{\max } \Lambda_{t}^{\prime \nu}$. Therefore $\Lambda_{t}^{\prime \nu}<\alpha_{l}^{\bar{c}_{t}^{\prime \nu}} \Leftrightarrow \alpha_{l}^{\bar{c}_{t}^{\prime \nu}}>\frac{p_{t} \bar{c}_{t}^{\prime \nu}-\left(1+r_{t}-R_{t}\right) W_{t}^{\prime \nu}}{w_{t}^{\max }}$ and the desired inequality follows by rearranging the latter expression and noting that $1+r_{t}-R_{t}>0$.

A similar argument proves that (6) also holds.

Theorem 1 and Lemma 4 provide two different partitions of the set of countries, according to their UE exploitation or class status. Depending on the UE definition that one adopts, the two partitions may or may not coincide. Yet, an important intuition of UE theory is the existence of a robust relation between class and UE exploitation status. Based on Roemer [21], we formulate this intuition explicitly as follows:

Class-Exploitation Correspondence Principle $(\mathbf{C E C P}):$ Let $\left((\mathbf{p}, \mathbf{r}),\left(\xi^{\nu}\right)_{\nu \in \mathcal{N}}\right)$ be an 
IRS for $E\left(\Omega_{0}\right)$ such that $1+r_{t}-R_{t}>0$ for all $t$. Then, at all $t$

$$
\begin{aligned}
& \nu \in C_{t}^{1} \Rightarrow \nu \in \mathcal{N}_{t}^{t e r} \\
& \nu \in C_{t}^{3} \cup C_{t}^{4} \Rightarrow \nu \in \mathcal{N}_{t}^{t e d} .
\end{aligned}
$$

CECP states that in equilibrium, countries in the upper classes in the credit market should emerge as UE exploiters, while those in the lower classes should be UE exploited. Theorem 2 provides necessary and sufficient conditions for a UE definition in the admissible domain to satisfy both WECP and CECP:

Theorem 2 (CECP): Let $\left((\mathbf{p}, \mathbf{r}),\left(\xi^{\nu}\right)_{\nu \in \mathcal{N}}\right)$ be an IRS for $E\left(P, \mathcal{N}, u, \rho, \Omega_{0}\right)$ such that $1+$ $r_{t}>\max _{i} \frac{p_{i t}}{p_{i t-1}}$ for all $t$. Given any definition of UE exploitation satisfying $\mathbf{L E}$, the following statements are equivalent:

(i) WECP and CECP hold;

(ii) at all $t$, there exist $\bar{W}_{t}, \underline{W}_{t}>0$ with $p_{t-1} \underline{\alpha}_{t}^{\min } \leqq \underline{W}_{t} \leqq \bar{W}_{t} \leqq p_{t-1} \underline{\alpha}_{t}^{\max }$ such that for any $E\left(P, \mathcal{N}, u, \rho, \Omega_{0}^{\prime}\right) \in \mathcal{E}$ with $\sum_{\nu \in \mathcal{N}} \omega_{0}^{\prime \nu}=\sum_{\nu \in \mathcal{N}} \omega_{0}^{\nu}$ and any $\operatorname{IRS}\left((\mathbf{p}, \mathbf{r}),\left(\xi^{\prime \nu}\right)_{\nu \in \mathcal{N}}\right)$ for $E\left(P, \mathcal{N}, u, \rho, \Omega_{0}^{\prime}\right)$ with $\sum_{\nu \in \mathcal{N}} \omega_{t}^{\prime \nu}=\sum_{\nu \in \mathcal{N}} \omega_{t}^{\nu}$, all $t$, and for each $\nu \in \mathcal{N}$,

$$
\begin{aligned}
W_{t}^{\prime \nu}>\bar{W}_{t} \Leftrightarrow W_{t}^{\prime \nu}>\frac{p_{t} \bar{c}_{t}^{\prime \nu}-w_{t}^{\max } \alpha_{l}^{\bar{c}_{t}^{\prime \nu}}}{1+r_{t}-R_{t}} ; \\
W_{t}^{\prime \nu}<\underline{W}_{t} \Leftrightarrow W_{t}^{\prime \nu}<\frac{p_{t} \underline{c}_{t}^{\prime \nu}-w_{t}^{\max } \alpha_{l}^{\underline{c}_{t}^{\prime \nu}}}{1+r_{t}-R_{t}} .
\end{aligned}
$$

Proof: 1. Consider any economy $E\left(P, \mathcal{N}, u, \rho, \Omega_{0}^{\prime}\right) \in \mathcal{E}$ and any $\operatorname{IRS}\left((\mathbf{p}, \mathbf{r}),\left(\xi^{\prime \nu}\right)_{\nu \in \mathcal{N}}\right)$ for $E\left(P, \mathcal{N}, u, \rho, \Omega_{0}^{\prime}\right)$. Note that $1+r_{t}>\max _{i} \frac{p_{i t}}{p_{i t-1}}$ for all $t$ implies $1+r_{t}-R_{t}>0$ for all $t$. Given a definition of exploitation satisfying $\mathbf{L E}$, at any $t$, for each $\nu \in \mathcal{N}$, there exist $\bar{c}_{t}^{\prime \nu}, \underline{c}_{t}^{\prime \nu} \in B_{t}\left((\mathbf{p}, \mathbf{r}) ; W_{t}^{\prime \nu}, \Lambda_{t}^{\prime \nu}\right)$ such that there exist $\alpha^{\bar{c}_{t}^{\prime \nu}} \in \psi\left(\bar{c}_{t}^{\prime \nu}\right) \cap \partial P$ with $p_{t} \widehat{\alpha}^{c_{t}^{\prime \nu}} \geqq p_{t} \bar{c}_{t}^{\prime \nu}$ and $\alpha^{\underline{c}_{t}^{\prime \nu}} \in \psi\left(\underline{c}_{t}^{\prime \nu}\right) \cap \partial P$ with $p_{t} \widehat{\alpha}_{t}^{\underline{c}_{t}^{\prime \nu}} \geqq p_{t} \underline{t}_{t}^{\prime \nu}$ satisfying: $\alpha_{l}^{\underline{c}_{t}^{\prime \nu}} \geqq \alpha_{l}^{\bar{c}_{t}^{\prime \nu}}$, and $\nu \in \mathcal{N}_{t}^{t e r} \Leftrightarrow \Lambda_{t}^{\prime \nu}<\alpha_{l}^{\bar{c}_{t}^{\prime \nu}}$ and $\nu \in \mathcal{N}_{t}^{t e d} \Leftrightarrow \Lambda_{t}^{\prime \nu}>\alpha_{l}^{\underline{c}_{t}^{\prime \nu}}$.

2. ((ii) $\Rightarrow(\mathrm{i}))$ Suppose that (ii) holds. Then by Lemma 4 and Theorem 1 it immediately follows that WECP and CECP hold. 
3. ((i) $\Rightarrow($ ii)) Let WECP and CECP hold. By Lemma 4, it is sufficient to show that at all $t, \bar{W}_{t} \leqq p_{t-1} \underline{\alpha}_{t}^{\max }$ and $p_{t-1} \underline{\alpha}_{t}^{\min } \leqq \underline{W}_{t}$.

Suppose, by way of contradiction, that $\bar{W}_{t}>p_{t-1} \underline{\alpha}_{t}^{\max }$, some $t$. We consider two cases.

Case 1: suppose that $p_{t-1} \underline{\alpha}_{t}^{\max } \geqq p_{t-1} \omega_{t}^{\prime \nu}$, all $\nu \in \mathcal{N}$, for any $E\left(P, \mathcal{N}, u, \rho, \Omega_{0}^{\prime}\right) \in \mathcal{E}$ with $\sum_{\nu \in \mathcal{N}} \omega_{0}^{\prime \nu}=\sum_{\nu \in \mathcal{N}} \omega_{0}^{\nu}$ and any $\operatorname{IRS}\left((\mathbf{p}, \mathbf{r}),\left(\xi^{\prime \nu}\right)_{\nu \in \mathcal{N}}\right)$ for $E\left(P, \mathcal{N}, u, \rho, \Omega_{0}^{\prime}\right)$ with $\sum_{\nu \in \mathcal{N}} \omega_{t}^{\prime \nu}=$ $\sum_{\nu \in \mathcal{N}} \omega_{t}^{\nu}$, all $t$. By Theorem $1, C_{t}^{1}=\varnothing$. Moreover, because $\bar{W}_{t}>p_{t-1} \underline{\alpha}_{t}^{\max }$, by WECP $\mathcal{N}_{t}^{t e r}=\varnothing$. But then, noting that the same holds for any $\bar{W}_{t}^{\prime} \geqq p_{t-1} \underline{\alpha}_{t}^{\max }$ and that WECP does not require wealth thresholds to be unique, it is possible to set $\bar{W}_{t}=p_{t-1} \underline{\alpha}_{t}^{\max }$

Case 2: suppose that there exists an economy $E\left(P, \mathcal{N}, u, \rho, \Omega_{0}^{\prime}\right) \in \mathcal{E}$ with $\sum_{\nu \in \mathcal{N}} \omega_{0}^{\prime \nu}=$ $\sum_{\nu \in \mathcal{N}} \omega_{0}^{\nu}$ and an IRS $\left((\mathbf{p}, \mathbf{r}),\left(\xi^{\prime \nu}\right)_{\nu \in \mathcal{N}}\right)$ for $E\left(P, \mathcal{N}, u, \rho, \Omega_{0}^{\prime}\right)$ with $\sum_{\nu \in \mathcal{N}} \omega_{t}^{\prime \nu}=\sum_{\nu \in \mathcal{N}} \omega_{t}^{\nu}$, all $t$, such that $p_{t-1} \underline{\alpha}_{t}^{\max }<p_{t-1} \omega_{t}^{\nu}$, for some $\nu \in \mathcal{N}$. If $p_{t-1} \underline{\alpha}_{t}^{\max }<p_{t-1} \omega_{t}^{\prime \nu} \leqq \bar{W}_{t}$, then the desired contradiction follows from Theorem 1, CECP, and WECP. So, suppose that $p_{t-1} \underline{\alpha}_{t}^{\max }<\bar{W}_{t}<p_{t-1} \omega_{t}^{\prime \nu}$. Then by Lemma 2 it is immediate to show that there exists another economy $E\left(P, \mathcal{N}, u, \rho, \Omega_{0}^{\prime \prime}\right) \in \mathcal{E}$ with $\sum_{\nu \in \mathcal{N}} \omega_{0}^{\prime \prime \nu}=\sum_{\nu \in \mathcal{N}} \omega_{0}^{\nu}$ and an $\operatorname{IRS}\left((\mathbf{p}, \mathbf{r}),\left(\xi^{\prime \prime \nu}\right)_{\nu \in \mathcal{N}}\right)$ for $E\left(P, \mathcal{N}, u, \rho, \Omega_{0}^{\prime \prime}\right)$ with $\sum_{\nu \in \mathcal{N}} \omega_{t}^{\prime \prime \nu}=\sum_{\nu \in \mathcal{N}} \omega_{t}^{\nu}$, all $t$, such that $p_{t-1} \underline{\alpha}_{t}^{\max }<p_{t-1} \omega_{t}^{\prime \prime \nu} \leqq \bar{W}_{t}$ for some $\nu \in \mathcal{N}$, which yields the desired contradiction.

A similar argument can be used to prove that $p_{t-1} \underline{\alpha}_{t}^{\min } \leqq \underline{W}_{t}$.

These results fully characterise exploitative international relations in the intertemporal model. Theorem 1 identifies the structure of the global capital market, in which developed countries emerge as net lenders and less developed countries as net borrowers. Lemma 4 derives necessary and sufficient conditions for exploitative international relations to map inequalities in economic development (proxied by the value of productive endowments). Finally, Theorem 2 provides necessary and sufficient conditions for class positions in the global credit market to map wealth inequalities and exploitation status.

It is worth stressing at this point the generality of our results, which are derived in intertemporal economies, under rather general assumptions on preferences and technology, and without restricting the analysis to steady states. Perhaps more importantly, they are derived without adopting any specific UE approach: they hold for every definition within 
the admissible domain identified by $\mathbf{L E}$. The relation between wealth, class and exploitation is thus proved to hold for an entire (and potentially large) class of UE definitions.

\section{A Definition of UE Exploitation}

Section 4 provides a complete characterisation of the class of UE definitions that satisfy LE, WECP and CECP. But are there any definitions that actually meet the conditions in Theorem 2? This is not an idle question. Yoshihara [31] has shown that in static economies with revenue-maximising agents, some of the received definitions - including Morishima's [17] and Roemer's [21] - satisfy LE but not CECP. Roemer [21, 23] himself has raised doubts on the robustness of the relation between wealth, exploitation, and class. In this section, we show that the class of definitions identified by Theorem 2 is nonempty. To this end, we introduce a dynamic generalisation of a definition recently proposed by Yoshihara and Veneziani $[33,31,29]$ and show that it satisfies LE and preserves WECP and CECP, in the international economies considered in this paper.

Let $\left((\mathbf{p}, \mathbf{r}),\left(\xi^{\nu}\right)_{\nu \in \mathcal{N}}\right)$ be a RS for $E\left(\Omega_{0}\right)$ and let $\alpha_{t}^{\mathbf{p}, \mathbf{r}}+\beta_{t}^{\mathbf{p}, \mathbf{r}}$ denote the aggregate equilibrium production activity at $t$. For any $c \in \mathbb{R}_{+}^{n}$, such that $p_{t} c \leqq p_{t}\left(\widehat{\alpha}_{t}^{\mathbf{p}, \mathbf{r}}+\widehat{\beta}_{t}^{\mathbf{p}, \mathbf{r}}\right)$, the labour content of $c$ is equal to $\tau_{t}^{c}\left(\alpha_{l t}^{\mathbf{p}, \mathbf{r}}+\beta_{l t}^{\mathbf{p}, \mathbf{r}}\right)$, where $\tau_{t}^{c} \in[0,1]$ is such that $\tau_{t}^{c} p_{t}\left(\widehat{\alpha}_{t}^{\mathbf{p}, \mathbf{r}}+\widehat{\beta}_{t}^{\mathbf{p}, \mathbf{r}}\right)=$ $p_{t} c{ }^{20}$ Thus, the labour contained in any bundle $c$ (whose value does not exceed global income) is equal to the fraction $\tau^{c}$ of social labour, $\tau^{c}\left(\alpha_{l t}^{\mathbf{p}, \mathbf{r}}+\beta_{l t}^{\mathbf{p}, \mathbf{r}}\right)$, necessary to produce a fraction of aggregate net output, $\tau^{c}\left(\widehat{\alpha}_{t}^{\mathbf{p}, \mathbf{r}}+\widehat{\beta}_{t}^{\mathbf{p}, \mathbf{r}}\right)$, that has the same value as $c$. We denote this as l.v. $\left(c ;(\mathbf{p}, \mathbf{r}), \alpha_{t}^{\mathbf{p}, \mathbf{r}}+\beta_{t}^{\mathbf{p}, \mathbf{r}}\right)$ : it is the labour value of $c$ at $t$, at a RS with prices $(\mathbf{p}, \mathbf{r})$ and aggregate production, $\alpha_{t}^{\mathbf{p}, \mathbf{r}}+\beta_{t}^{\mathbf{p}, \mathbf{r}}$. Then:

Definition 4: Consider any economy $E\left(\Omega_{0}\right) \in \mathcal{E}$. Let $\left((\mathbf{p}, \mathbf{r}),\left(\xi^{\nu}\right)_{\nu \in \mathcal{N}}\right)$ be a RS for $E\left(\Omega_{0}\right)$. At all $t$, country $\nu \in \mathcal{N}$, which supplies $\Lambda_{t}^{\nu}$, is $W P_{t^{-}}$exploited if and only if $\Lambda_{t}^{\nu}>$ l.v. $\left(\widetilde{c}_{t}^{\nu} ;(\mathbf{p}, \mathbf{r}), \alpha_{t}^{\mathbf{p}, \mathbf{r}}+\beta_{t}^{\mathbf{p}, \mathbf{r}}\right)$ for any $\widetilde{c}_{t}^{\nu} \in B_{t}\left((\mathbf{p}, \mathbf{r}) ; p_{t-1} \omega_{t}^{\nu}, \Lambda_{t}^{\nu}\right)$, and a $W P_{t^{-}}$exploiter if and only if $\Lambda_{t}^{\nu}<l . v \cdot\left(\widetilde{c}_{t}^{\nu} ;(\mathbf{p}, \mathbf{r}), \alpha_{t}^{\mathbf{p}, \mathbf{r}}+\beta_{t}^{\mathbf{p}, \mathbf{r}}\right)$ for any $\widetilde{c}_{t}^{\nu} \in B_{t}\left((\mathbf{p}, \mathbf{r}) ; p_{t-1} \omega_{t}^{\nu}, \Lambda_{t}^{\nu}\right)$.

\footnotetext{
${ }^{20}$ If $p_{t}\left(\widehat{\alpha}_{t}^{\mathbf{p}, \mathbf{r}}+\widehat{\beta}_{t}^{\mathbf{p}, \mathbf{r}}\right)=0$, we set $\tau_{t}^{c}=0$ by definition.
} 
Definition 4 generalises the definition proposed by Yoshihara and Veneziani [33, 31, 29] in a static context. In static economies, exploitation status is measured with reference to consumption bundles that agents can purchase with their actual net income. As argued in section 4.1 above, however, in the general dynamic framework considered here, actual net income is not necessarily a normatively appropriate variable to determine agents' exploitation status and we propose to focus instead on the net income that could be devoted to consumption if an agent decided only to replace its wealth.

Definition 4 is conceptually related to the 'New Interpretation' (Duménil [6, 7]; Foley [12]; Duménil et al [8]). In fact, $\tau^{\widetilde{c}_{t}^{\nu}}$ is $\nu$ 's reference share of world income, and so $\tau^{\widetilde{c}_{t}^{\nu}}\left(\alpha_{l t}^{\mathbf{p}, \mathbf{r}}+\beta_{l t}^{\mathbf{p}, \mathbf{r}}\right)$ is the share of total social labour that $\nu$ receives by earning national income $p_{t} \widetilde{c}_{t}^{\nu}$. Then, as in the New Interpretation, country $\nu$ is UE exploited if and only if the amount of social labour it receives is less than the amount of labour expended by its workers, $\Lambda_{t}^{\nu}$.

Several attractive features of Definition 4 are worth noting. First, it does not rely on the labour theory of value and is not restricted to economies with a linear technology. Second, unlike in the standard approach, exploitation is not a merely technological phenomenon and social relations play a central role. For in Definition 4 the definition of UE exploitation requires knowledge of equilibrium prices and of the social reproduction point, and it is related to the production and distribution of global income and social labour. Third, UE exploitation is identified as a feature of the competitive allocation of social labour rather than as the result of productive inefficiencies, or labour market imperfections. Fourth, Definition 4 transparently captures the key intuitions of UE theory. For it identifies exploitation status by comparing the labour contributed by each country $\nu$ and the share of aggregate social labour received by $\nu$ via its national income. Moreover, Yoshihara and Veneziani [33] have shown that in a rich domain of (static) convex economies, Definition 4 is the only UE definition that satisfies a small set of formally weak and theoretically desirable properties.

Theorem 3 proves that if Definition 4 is adopted then both WECP and CECP hold.

Theorem 3: Let $\left((\mathbf{p}, \mathbf{r}),\left(\xi^{\nu}\right)_{\nu \in \mathcal{N}}\right)$ be an IRS for $E\left(\Omega_{0}\right)$ such that $1+r_{t}>\max _{i} \frac{p_{i t}}{p_{i t-1}}$ for all t. Then, under Definition 4, WECP and CECP hold. 
Proof: 1. First, we show that Definition 4 satisfies $\mathbf{L E}$ at an IRS. Since $\left((\mathbf{p}, \mathbf{r}),\left(\xi^{\nu}\right)_{\nu \in \mathcal{N}}\right)$ is an IRS for $E\left(\Omega_{0}\right)$, it follows that $\left(\alpha_{t}^{\mathbf{p}, \mathbf{r}}+\beta_{t}^{\mathbf{p}, \mathbf{r}}\right) \in P_{t}^{w}(\mathbf{p}, \mathbf{r})$ and $\underline{\alpha}_{t}^{\mathbf{p}, \mathbf{r}}+\underline{\beta}_{t}^{\mathbf{p}, \mathbf{r}}=\omega_{t}$, at all $t$. Further, by Lemma $2, \Lambda_{t}^{\nu}=\Lambda_{t}^{*}$ for all $t$ and all $\nu \in \mathcal{N}$. At all $t$, let $\left(\theta_{t}^{\nu}\right)_{\nu \in \mathcal{N}} \in[0,1]^{N}$ be such that $\sum_{\nu \in \mathcal{N}} \theta_{t}^{\nu}=1$ and $p_{t-1} \omega_{t}^{\nu}=\theta_{t}^{\nu} p_{t-1} \omega_{t}$ for each $\nu \in \mathcal{N}$. Then, at all $t$, and for all $\nu \in \mathcal{N}$

$$
\begin{aligned}
& \left(1+r_{t}\right) p_{t-1} \omega_{t}^{\nu}+w_{t}^{\max } \Lambda_{t}^{*}-R_{t} p_{t-1} \omega_{t}^{\nu} \\
& =\left(1+r_{t}-R_{t}\right) \theta_{t}^{\nu} p_{t-1} \omega_{t}+w_{t}^{\max } \Lambda_{t}^{*} \\
& =\theta_{t}^{\nu}\left[p_{t}\left(\widehat{\alpha}_{t}^{\mathbf{p}, \mathbf{r}}+\widehat{\beta}_{t}^{\mathbf{p}, \mathbf{r}}\right)-w_{t}^{\max }\left(\alpha_{l t}^{\mathbf{p}, \mathbf{r}}+\beta_{l t}^{\mathbf{p}, \mathbf{r}}\right)\right]+w_{t}^{\max } \Lambda_{t}^{*}
\end{aligned}
$$

Then, because $1+r_{t}-R_{t}>0$ and $w_{t}^{\max }>0$, all $t$, in each period $t$, there exists $\left(\tau_{t}^{\nu}\right)_{\nu \in \mathcal{N}} \in$ $(0,1)^{N}$ such that $\sum_{\nu \in \mathcal{N}} \tau_{t}^{\nu}=1$ and

$$
\tau_{t}^{\nu} p_{t}\left(\widehat{\alpha}_{t}^{\mathbf{p}, \mathbf{r}}+\widehat{\beta}_{t}^{\mathbf{p}, \mathbf{r}}\right)=\theta_{t}^{\nu}\left[p_{t}\left(\widehat{\alpha}_{t}^{\mathbf{p}, \mathbf{r}}+\widehat{\beta}_{t}^{\mathbf{p}, \mathbf{r}}\right)-w_{t}^{\max }\left(\alpha_{l t}^{\mathbf{p}, \mathbf{r}}+\beta_{l t}^{\mathbf{p}, \mathbf{r}}\right)\right]+w_{t}^{\max } \Lambda_{t}^{*}
$$

According to Definition 4, at all $t, \nu \in \mathcal{N}_{t}^{t e r}$ if and only if $\Lambda_{t}^{*}<\tau_{t}^{\nu}\left(\alpha_{l t}^{\mathbf{p}, \mathbf{r}}+\beta_{l t}^{\mathbf{p}, \mathbf{r}}\right)$; and $\nu \in \mathcal{N}_{t}^{t e d}$ if and only if $\Lambda_{t}^{*}>\tau_{t}^{\nu}\left(\alpha_{l t}^{\mathbf{p}, \mathbf{r}}+\beta_{l t}^{\mathbf{p}, \mathbf{r}}\right)$. By taking $\bar{c}_{t}^{\nu}=\underline{c}_{t}^{\nu}=\tau_{t}^{\nu} \frac{p_{t}\left(\widehat{\alpha}_{t}^{\mathbf{p}, \mathbf{r}}+\widehat{\beta}_{t}^{\mathbf{p}, \mathbf{r}}\right)}{p_{t}\left(\bar{\alpha}_{t}^{\mathbf{p}, \mathbf{r}}+\bar{\beta}_{t}^{\mathbf{p}, \mathbf{r}}\right)}\left(\bar{\alpha}_{t}^{\mathbf{p}, \mathbf{r}}+\bar{\beta}_{t}^{\mathbf{p}, \mathbf{r}}\right)$ and $\alpha^{\bar{c}_{t}^{\nu}}=\alpha^{\underline{c}_{t}^{\nu}}=\tau_{t}^{\nu}\left(\alpha_{t}^{\mathbf{p}, \mathbf{r}}+\beta_{t}^{\mathbf{p}, \mathbf{r}}\right)$, for all $\nu \in \mathcal{N}$, we can see that Definition 4 satisfies $\mathbf{L E}$.

2. By step 1, it suffices to show that under Definition 4, statement (ii) of Theorem 2 holds. Let $\left((\mathbf{p}, \mathbf{r}),\left(\xi^{\nu}\right)_{\nu \in \mathcal{N}}\right)$ be an IRS for $E\left(P, \mathcal{N}, u, \rho, \Omega_{0}\right)$ such that $1+r_{t}>\max _{i} \frac{p_{i t}}{p_{i t-1}}$ for all $t$. We show that at all $t, \bar{W}_{t}=\underline{W}_{t}=W_{t}^{*} \equiv \frac{1}{N} p_{t-1} \omega_{t}>0$ satisfies all conditions in statement (ii).

First of all, note that for any $E\left(P, \mathcal{N}, u, \rho, \Omega_{0}^{\prime}\right) \in \mathcal{E}$ with $\sum_{\nu \in \mathcal{N}} \omega_{0}^{\prime \nu}=\sum_{\nu \in \mathcal{N}} \omega_{0}^{\nu}$ and any IRS $\left((\mathbf{p}, \mathbf{r}),\left(\xi^{\prime \nu}\right)_{\nu \in \mathcal{N}}\right)$ for $E\left(P, \mathcal{N}, u, \rho, \Omega_{0}^{\prime}\right)$ with $\sum_{\nu \in \mathcal{N}} \omega_{t}^{\prime \nu}=\sum_{\nu \in \mathcal{N}} \omega_{t}^{\nu}$, all $t, W_{t}^{*}$ is well defined, unique and invariant.

Further, for any $E\left(P, \mathcal{N}, u, \rho, \Omega_{0}^{\prime}\right) \in \mathcal{E}$ with $\sum_{\nu \in \mathcal{N}} \omega_{0}^{\prime \nu}=\sum_{\nu \in \mathcal{N}} \omega_{0}^{\nu}$ and any $\operatorname{IRS}\left((\mathbf{p}, \mathbf{r}),\left(\xi^{\prime \nu}\right)_{\nu \in \mathcal{N}}\right)$ 
for $E\left(P, \mathcal{N}, u, \rho, \Omega_{0}^{\prime}\right)$ with $\sum_{\nu \in \mathcal{N}} \omega_{t}^{\prime \nu}=\sum_{\nu \in \mathcal{N}} \omega_{t}^{\nu}$, all $t$, for each $\nu \in \mathcal{N}$, we have

$$
\begin{aligned}
p_{t} \widehat{\alpha}^{\bar{c}_{t}^{\prime \nu}}-w_{t}^{\max } \alpha_{l}^{\bar{c}_{t}^{\prime \nu}} & =\tau_{t}^{\prime \nu}\left[p_{t}\left(\widehat{\alpha}_{t}^{\prime \mathbf{p}, \mathbf{r}}+\widehat{\beta}_{t}^{\prime \mathbf{p}, \mathbf{r}}\right)-w_{t}^{\max }\left(\alpha_{l t}^{\prime \mathbf{p}, \mathbf{r}}+\beta_{l t}^{\prime \mathbf{p}, \mathbf{r}}\right)\right] \\
& =\tau_{t}^{\prime \nu}\left[\left(1+r_{t}\right) p_{t-1}\left(\underline{\alpha}_{t}^{\prime \mathbf{p}, \mathbf{r}}+\underline{\beta}_{t}^{\prime \mathbf{p}, \mathbf{r}}\right)-p_{t}\left(\underline{\alpha}_{t}^{\prime \mathbf{p}, \mathbf{r}}+\underline{\beta}_{t}^{\prime \mathbf{p}, \mathbf{r}}\right)\right] \\
& =\tau_{t}^{\prime \nu}\left(1+r_{t}-R_{t}\right) p_{t-1} \omega_{t}
\end{aligned}
$$

where the first equality follows from step 1, the second equality follows from the fact that $\left(\alpha_{t}^{\prime \mathbf{p}, \mathbf{r}}+\beta_{t}^{\mathbf{p}, \mathbf{r}}\right) \in P_{t}^{w}(\mathbf{p}, \mathbf{r})$ at a RS, and the last equality follows from the definition of $R_{t}$ noting that at a RS $\underline{\alpha}_{t}^{\prime \mathbf{p}, \mathbf{r}}+\underline{\beta}_{t}^{\mathbf{p}, \mathbf{r}}=\omega_{t}$. Then, since $p_{t} \widehat{\alpha}^{\bar{c}_{t}^{\prime \nu}}=p_{t} \bar{c}_{t}^{\prime \nu}=p_{t} \widehat{\alpha}^{\underline{c}_{t}^{\prime \nu}}=p_{t} \underline{c}_{t}^{\prime \nu}$ and $\alpha^{\bar{c}_{t}^{\prime \nu}}=\alpha_{c_{t}^{\prime \prime}}^{c^{\prime \prime}}$ by step 1 , it follows that for any $E\left(P, \mathcal{N}, u, \rho, \Omega_{0}^{\prime}\right) \in \mathcal{E}$ with $\sum_{\nu \in \mathcal{N}} \omega_{0}^{\prime \nu}=\sum_{\nu \in \mathcal{N}} \omega_{0}^{\nu}$ and any $\operatorname{IRS}\left((\mathbf{p}, \mathbf{r}),\left(\xi^{\prime \nu}\right)_{\nu \in \mathcal{N}}\right)$ for $E\left(P, \mathcal{N}, u, \rho, \Omega_{0}^{\prime}\right)$ with $\sum_{\nu \in \mathcal{N}} \omega_{t}^{\prime \nu}=\sum_{\nu \in \mathcal{N}} \omega_{t}^{\nu}$, all $t$, for each $\nu \in \mathcal{N}$,

$$
\begin{aligned}
W_{t}^{\prime \nu}>W_{t}^{*} \Leftrightarrow W_{t}^{\prime \nu}>\frac{p_{t} \bar{c}_{t}^{\prime \nu}-w_{t}^{\max } \alpha_{l}^{\bar{\tau}_{t}^{\prime \nu}}}{1+r_{t}-R_{t}} ; \\
W_{t}^{\prime \nu}<W_{t}^{*} \Leftrightarrow W_{t}^{\prime \nu}<\frac{p_{t} \underline{c}_{t}^{\prime \nu}-w_{t}^{\max } \alpha_{l}^{c_{t}^{\prime \nu}}}{1+r_{t}-R_{t}} .
\end{aligned}
$$

Finally, since $W_{t}^{*}=\frac{1}{N} p_{t-1} \omega_{t}=p_{t-1}\left(\frac{\underline{\alpha}_{t}^{\prime \mathbf{p}, \mathbf{r}}+\underline{\beta}_{t}^{\prime \mathbf{p}, \mathbf{r}}}{N}\right),\left(\alpha_{t}^{\prime \mathbf{p}, \mathbf{r}}+\beta_{t}^{\prime \mathbf{p}, \mathbf{r}}\right) \in P_{t}^{w}(\mathbf{p}, \mathbf{r})$ implies that $p_{t-1} \underline{\alpha}_{t}^{\min } \leqq W_{t}^{*} \leqq p_{t-1} \underline{\alpha}_{t}^{\max }$.

In summary, statement (ii) of Theorem 2 holds under Definition 4 and therefore WECP and CECP hold under Definition 4.

Theorem 3 implies that the set of definitions identified in Theorem 2 is nonempty. If Definition 4 is adopted, then both Roemer's [21] Class-Exploitation Correspondence Principle and the Wealth-Exploitation Correspondence Principle can be extended to general, dynamic equilibrium paths of international economies with general convex technologies and welfare functions. Theorems 1 and 3 allow us to identify the structure of dependent and exploitative international relations emerging between developed and less developed countries as the equilibrium outcome of a perfectly competitive international economy. Mutual benefits from free international trade of commodities and capital coexist with an international stratification of 
countries in the credit market and with unequal flows of revenue and labour.

\section{Conclusion}

This paper develops an axiomatic analysis of the concept of unequal exchange between countries in a dynamic general equilibrium model, which generalises Roemer's [21, 22] economy with a global capital market. The class of definitions that preserve three fundamental properties of UE exploitation theory - including the existence of a correspondence between wealth, class and exploitation status, - in general dynamic equilibria is completely characterised. This class is shown to be nonempty: there exists a UE definition that satisfies a basic domain axiom and both the Wealth-Exploitation Correspondence Principle and the Class-Exploitation Correspondence Principle. This definition is conceptually related to the so-called 'New Interpretation' (Duménil [6, 7]; Foley [12]; Duménil et al [8]). It is logically consistent, general, and firmly anchored to empirically observed data.

Based on this definition, unequal international relations are fully characterised and Roemer's $[21,22]$ results generalised. In equilibrium, countries are partitioned based on their UE exploitation status and on their position in the capital market: advanced countries are net lenders and exploiters, less developed countries are net borrowers and suffer from UE exploitation. Mutual gains from trade and UE exploitation coexist in competitive markets. The exploitative nature of international relations is the product of capital flows, which transfer surplus from less developed to more developed countries.

It may be objected that in reality capital flows do not move in the direction predicted in Theorem 1: as Lucas [14] famously argued, capital does not flow to underdeveloped countries. Two points should be made here that suggest that the so-called "Lucas paradox" does not pose a major challenge to our conclusions. Firstly, there is a growing empirical literature suggesting that there may be no paradox after all: the actual pattern of credit flows may be explained by some fundamental violations of the basic assumptions of the neoclassical model which are also shared by Roemer's [21, 22] model and our extension of it, such as differences in fundamentals, including institutional quality (Alfaro et al [1]), and in credit 
risk (Reinhart and Rogoff [18]). Actually, Reinhardt et al ([19], pp.235-6) have shown that "the prediction of the standard neoclassical theory holds only when taking into account the degree of capital account openness, conditional on a set of fundamentals. Among countries with an open capital account, richer countries tend to experience net capital outflows, while poorer countries tend to experience net capital inflows. In contrast, in countries with closed capital account, there appears to be no systematic relationship between the level of economic development and net capital flows." According to them, the phenomenon observed by Lucas [14] is due to the relatively high degree of capital controls in the period he considered. The paradox, however, disappeared in the 1990s when capital controls were lifted and "this liberalization process was associated with significant changes in the patterns of capital flows across countries at different income levels" (Reinhardt et al [19], p.236). ${ }^{21}$

Secondly, and perhaps more importantly, ours is not an investigation of the determinants of the size and direction of capital flows in the current global economy. This paper develops a normative, axiomatic analysis of the concept of exploitation and for this purpose we have followed Roemer ([22], p.58) in using Occam's razor and abstracting from "many elements of friction or noncompetitiveness or "imperfections"'. These simplifying assumptions are theoretically appropriate from a normative perspective, as they allow us to derive precise conclusions on the concept of UE in an abstract and general, but at the same time well-defined context, focusing on a benchmark competitive economy. Our main axioms are thus restricted to hold at the competitive equilibria of the intertemporal economy with unrestricted capital flows and no labour mobility - the "classical [UE] environment" (Roemer [22], p.53). The appropriate extension of our axiomatic analysis to noncompetitive settings and disequilibrium allocations is an interesting and open question.

Our analysis thus provides a normative benchmark to evaluate international relations under globalisation. For, inequalities in wealth and development among countries are at least partly due to past "robbery and plunder" - especially during the colonial period -

\footnotetext{
${ }^{21}$ One may argue that such movements are still smaller than predicted by the standard neoclassical model based on international differences in the marginal product of capital. As Lucas ([14], p.92) put it, "one would expect no investment to occur in the wealthy countries in the face of return differentials of this magnitude." This issue is not really relevant in our framework, especially given that, as noted in section 3 , international capital flows are not determined by differences in the marginal productivity of capital.
} 
which makes them, and the unequal exchanges and exploitative relations resulting from them hardly justifiable, as argued by Ferguson and Veneziani [10]. To be sure, the radical change in ownership relations in the world economy necessary to eliminate UE exploitation may be considered politically infeasible. This does not make the concept of UE exploitation any less relevant. For it is essential to establish a robust normative benchmark against which to evaluate international relations, and even if it is not possible to eliminate UE exploitation in one stroke, there may be a number of measures to reduce it via international transfers and redistribution. An interesting question from this perspective concerns the development of a measure of the degree or intensity of UE exploitation of each country, and an index of aggregate UE exploitation in the international economy that goes beyond the rather coarse classification into UE exploiting and UE exploited nations. We leave this issue for further research.

\section{References}

[1] Alfaro, L., Kalemli-Ozcan, S., and V. Volosovych (2008): "Why Doesn't Capital Flow from Rich to Poor Countries? An Empirical Investigation," The Review of Economics and Statistics 90, 347-368.

[2] Amin, S. (1976): Unequal Development: An Essay on the Social Formations of Peripheral Capitalism, Monthly Review, New York.

[3] Bacha, E.L. (1978): "An Interpretation of Unequal Exchange from Prebisch-Singer to Emmanuel," Journal of Development Economics 5, 319-330.

[4] Ben-David, D. (1993): "Equalizing Exchange: Trade Liberalization and Income Convergence," Quarterly Journal of Economics 108, 653-679.

[5] Chen, Z. (1992): "Long-run Equilibria in a Dynamic Heckscher-Ohlin Model," Canadian Journal of Economics 92, 923-943.

[6] Duménil, G. (1980): De la Valeur aux Prix de Production, Economica, Paris. 
[7] Duménil, G. (1984): “The So-Called 'Transformation Problem' Revisited: A Brief Comment," Journal of Economic Theory 33, 340-348.

[8] Duménil, G., Foley, D.K., and D. Lévy (2009): "A Note on the Formal Treatment of Exploitation in a Model with Heterogeneous Labor," Metroeconomica 60, 560-567.

[9] Emmanuel, A. (1972): Unequal exchange, Monthly Review Press, New York.

[10] Ferguson, B. and R. Veneziani (2017): “Territorial Wrongs and Colonial Rights," mimeo, VU Amsterdam and Queen Mary University of London.

[11] Frank, A.G. (1978): Dependent accumulation and underdevelopment, Monthly Review Press, New York.

[12] Foley, D.K. (1982): "The Value of Money, the Value of Labor Power, and the Marxian Transformation Problem," Review of Radical Political Economics 14, 37-47.

[13] Griffin, K., and J. Gurley (1985): "Radical Analyses of Imperialism, The Third World, and the Transition to Socialism: A Survey Article," Journal of Economic Literature 23, 1089-1143.

[14] Lucas, R. (1990): “Why Doesn’t Capital Flow from Rich to Poor Countries?" American Economic Review 80, 92-96.

[15] Marx, K. (1968): Theories of Surplus Value, Vol.III, Lawrence \& Wishart, London.

[16] Morishima, M. (1969): Theory of Economic Growth, Oxford University Press, Oxford.

[17] Morishima, M. (1974): "Marx in the Light of Modern Economic Theory," Econometrica 42, 611-632.

[18] Reinhart, C.M. and K.S. Rogoff (2004): "Serial Default and the 'Paradox' of Rich-toPoor Capital Flows," American Economic Review 94, 53-58.

[19] Reinhardt, D., Ricci, L.A., and T. Tressel (2013): "International capital flows and development: Financial openness matters," Journal of International Economics 91, $235-251$. 
[20] Roemer, J.E. (1980): "A General Equilibrium Approach to Marxian Economics," Econometrica 48, 505-530.

[21] Roemer, J.E. (1982): A General Theory of Exploitation and Class, Harvard University Press, Cambridge.

[22] Roemer, J.E. (1983): "Unequal Exchange, Labor Migration, and International Capital Flows: A Theoretical Synthesis," in: Desai, P. (Ed.), Marxism, Central Planning and the Soviet Economy, MIT Press, Cambridge.

[23] Roemer, J.E. (1986): Value, exploitation, and class, Harwood Academic Publishers, New York.

[24] Roemer, J.E. and R. Veneziani (2004): "What we owe our children, they their children...," Journal of Public Economic Theory 6, 637-654.

[25] Silvestre, J. (2007): "Intergenerational equity and human development," in: Roemer, J. E. and K. Suzumura (Eds.), Intergenerational Equity and Sustainability, MacMillan, London.

[26] Slaughter, M.J. (2001): "Trade Liberalization and per capita Income Convergence: a Difference-in-differences Analysis," Journal of International Economics 55, 203-228.

[27] Veneziani, R. (2007): "Exploitation and Time," Journal of Economic Theory 132, 189207.

[28] Veneziani, R. (2013): "Exploitation, Inequality, and Power," Journal of Theoretical Politics 25, 526-545.

[29] Veneziani, R. and N. Yoshihara (2015): "Exploitation in Economies with Heterogeneous Preferences, Skills and Assets: An Axiomatic Approach," Journal of Theoretical Politics $27,8-33$.

[30] Veneziani, R. and N. Yoshihara (2017): "One million miles to go: taking the axiomatic road to defining exploitation," Cambridge Journal of Economics, forthcoming. 
[31] Yoshihara, N. (2010): "Class and Exploitation in General Convex Cone Economies," Journal of Economic Behavior \& Organization 75, 281-296.

[32] Yoshihara, N. (2017): "A Progress Report on Marxian Economic Theory: On the Controversies in Exploitation Theory since Okishio (1963)," Journal of Economic Surveys, forthcoming.

[33] Yoshihara, N. and R. Veneziani (2009): "Exploitation as the Unequal Exchange of Labour: An Axiomatic Approach," WP No.655, Queen Mary University of London.

[34] Yoshihara, N. and R. Veneziani (2011): "Strong Subjectivism in the Theory of Exploitation: A Critique," Metroeconomica 62, 53-68. 


\title{
Existence of an Interior Reproducible Solutions: Addendum to "Globalisation and Inequality in a Dynamic Economy: an Axiomatic Analysis of Unequal Exchange"
}

\author{
Roberto Veneziani*and Naoki Yoshihara ${ }^{\dagger}$
}

February 25, 2017

\begin{abstract}
This addendum proves the existence of an equilibrium for the economies analysed in the paper. The proof shows the consistency of the formal framework and establishes that the axioms in the paper do not hold vacuously.
\end{abstract}

*School of Economics and Finance, Queen Mary University of London, Mile End Road, London E1 4NS, UK. E-mail: r.veneziani@qmul.ac.uk

$\dagger$ (Corresponding author) Department of Economics, University of Massachusetts Amherst, Crotty Hall, 412 North Pleasant Street, Amherst, MA 01003, USA; The Institute of Economic Research, Hitotsubashi University, Kunitachi, Tokyo 186-0004, Japan; and School of Management, Kochi University of Technology, Kami-city, Kochi 782-8502, Japan. E-mail: n_yoshihara_1967@yahoo.co.jp 


\section{Existence of an Interior Reproducible So- lution}

In this Addendum, we prove the existence of an IRS in the economy $E\left(P, \mathcal{N}, u, \rho, \Omega_{0}\right)$ with $T=+\infty$. Similar arguments hold in the case with $T<+\infty$.

To begin with, consider the following social planner's optimisation programme $M P^{s p}$ :

$$
M P^{s p}: \max _{\left\{\left(c_{t}, \Lambda_{t}, \delta_{t}, \alpha_{t}, \omega_{t+1}\right)\right\}_{t=0}^{+\infty}} \sum_{t=0}^{+\infty} \rho^{t}\left(v\left(c_{t}\right)+N \phi\left(L-\Lambda_{t}\right)\right)
$$

subject to

$$
\begin{aligned}
\Lambda_{t} & \in[0, L],(\forall t) ; \\
N \Lambda_{t} & =\alpha_{l t},(\forall t) ; \\
\underline{\alpha}_{t}+\delta_{t} & \leqq \omega_{t},(\forall t) ; \\
\bar{\alpha}_{t}+\delta_{t} & \geqq c_{t}+\omega_{t+1},(\forall t) ; \\
\left(-\alpha_{l t},-\underline{\alpha}_{t}, \bar{\alpha}_{t}\right) & \in P,(\forall t), \\
\omega_{0} & \equiv \sum_{\nu \in \mathcal{N}} \omega_{0}^{\nu} .
\end{aligned}
$$

To solve $M P^{s p}$, we define the feasibility constraint correspondence $\Psi^{P}$ : $\mathbb{R}_{+}^{n} \rightarrow \mathbb{R}_{+}^{n}$ as:

$$
\begin{aligned}
& \Psi^{P}\left(\omega_{t}\right) \\
\equiv & \left\{\omega_{t+1} \in \mathbb{R}_{+}^{n} \mid \exists\left(c, \alpha_{t}\right) \in \mathbb{R}_{+}^{n} \times P: \alpha_{l t} \leqq N L, \underline{\alpha}_{t} \leqq \omega_{t}, \bar{\alpha}_{t}+\left(\omega_{t}-\underline{\alpha}_{t}\right) \geqq c+\omega_{t+1}\right\} .
\end{aligned}
$$

This correspondence is non-empty, compact-valued, and continuous. Let

$$
\Phi^{P}=\left\{\left(\omega_{t}, \omega_{t+1}\right) \in \mathbb{R}_{+}^{n} \times \mathbb{R}_{+}^{n} \mid \omega_{t+1} \in \Psi^{P}\left(\omega_{t}\right)\right\}
$$

be the graph of $\Psi^{P}$. For any given $\omega_{0}$, the set of feasible sequences is

$$
\mathcal{F}^{P}\left(\omega_{0}\right)=\left\{\left(\omega_{0}, \omega_{1}, \ldots, \omega_{t}, \ldots\right) \mid \omega_{t+1} \in \Psi^{P}\left(\omega_{t}\right) \text { for all } t\right\} .
$$


This correspondence is called the plan correspondence and it is non-empty. As in Aliprantis and Camera (2009, Theorems 2 and 3), the plan correspondence $\mathcal{F}^{P}$ can be proved to be non-empty, compact-valued, graph-closed, and continuous. For each $t$, for each $\left(\omega_{t}, \omega_{t+1}\right) \in \Phi^{P}$, let the one-period return function $F: \Phi^{P} \rightarrow \mathbb{R}$ be

$$
\begin{aligned}
F\left(\omega_{t}, \omega_{t+1}\right) & \equiv \max _{\left(c_{t}, \alpha_{t}\right) \in \mathbb{R}_{+}^{n} \times P} v\left(c_{t}\right)+N \phi\left(L-\Lambda_{t}\right) \\
\text { subject to } N \Lambda_{t} & =\alpha_{l t} \leqq N L, \underline{\alpha}_{t} \leqq \omega_{t}, \bar{\alpha}_{t}+\left(\omega_{t}-\underline{\alpha}_{t}\right) \geqq c_{t}+\omega_{t+1} .
\end{aligned}
$$

By Berge's Maximum Theorem, $F$ is continuous, as $\left\{\omega_{t}\right\} \times \Psi^{P}\left(\omega_{t}\right)$ is compactvalued and continuous. Then, $M P^{s p}$ reduces to the following programme $M P^{\text {sprd }}$ :

$$
\max _{\left(\omega_{0}, \omega_{1}, \ldots, \omega_{t}, \omega_{t+1}, \ldots\right) \in \mathcal{F}^{P}\left(\omega_{0}\right)} \sum_{t=0}^{+\infty} \rho^{t} F\left(\omega_{t}, \omega_{t+1}\right) .
$$

Following the arguments in Aliprantis and Camera (2009, Lemma 7(a)), the reduced social planner's programme $M P^{\text {sprd }}$ has an optimal solution $\left(\omega_{0}, \omega_{1}^{*}, \ldots, \omega_{t}^{*}, \omega_{t+1}^{*}, \ldots\right) \in \mathcal{F}^{P}\left(\omega_{0}\right)$. Then, correspondingly, there exists an optimal solution $\left\{\left(c_{t}^{*}, \Lambda_{t}^{*}, \delta_{t}^{*}, \alpha_{t}^{*}, \omega_{t+1}^{*}\right)\right\}_{t=0}^{+\infty}$ to the programme $M P^{s p}$. Let the set of optimal solutions to the programme $M P^{s p}$ be denoted by $\mathcal{O}\left(\omega_{0}\right)$.

Given the definition of IRS, we consider the following class of optimal solutions to programme $M P^{s p}$ :

Definition A.1: An non-trivial interior optimal solution to programme $M P^{s p}$ is an optimal solution $\left\{\left(c_{t}^{*}, \Lambda_{t}^{*}, \delta_{t}^{*}, \alpha_{t}^{*}, \omega_{t+1}^{*}\right)\right\}_{t=0}^{+\infty} \in \mathcal{O}\left(\omega_{0}\right)$ such that $c_{t}^{*}>\mathbf{0}, 0<\Lambda_{t}^{*}<L$, and $\bar{\alpha}_{t}^{*} \geq \mathbf{0}$ for all $t$.

By the assumptions on preferences and technology, it is not difficult to show that the set $O\left(\omega_{0}\right)$ contains non-trivial interior optimal solutions. ${ }^{1}$

Let $\left\{\left(c_{t}^{*}, \Lambda_{t}^{*}, \delta_{t}^{*}, \alpha_{t}^{*}, \omega_{t+1}^{*}\right)\right\}_{t=0}^{+\infty}$ be a non-trivial interior solution to programme $M P^{s p}$. Given that the production possibility set $P$ is convex and

\footnotetext{
${ }^{1}$ For instance, to ensure $\bar{\alpha}_{t}^{*} \geq \mathbf{0}$ for all $t$, assume on the contrary that $\bar{\alpha}_{t}^{*}=\mathbf{0}$ for some $t$. Then, $\alpha_{l t}^{*}=0=\Lambda_{t}^{*}$ and $\underline{\alpha}_{t}^{*}=\mathbf{0}$ must hold for the optimality. Then, $c_{t}^{*}+\omega_{t+1}^{*}=\omega_{t}^{*}$. However, by A2 and the cone property of $P$, there exists $\alpha^{\prime} \in P$ such that $\alpha_{l}^{\prime}>0$ is sufficiently small, $\underline{\alpha}^{\prime} \leqq \omega_{t}^{*}$, and $\bar{\alpha}^{\prime}+\left(\omega_{t}^{*}-\underline{\alpha}^{\prime}\right)>c_{t}^{*}+\omega_{t+1}^{*}$. Then, by the property of the function $\phi$, for a sufficiently small positive labour supply $\Lambda^{\prime}=\alpha_{l}^{\prime}$, it follows that $v\left(c^{\prime}\right)+\phi\left(\Lambda^{\prime}\right)>v\left(c_{t}^{*}\right)+\phi\left(\Lambda_{t}^{*}\right)$ for $c^{\prime} \equiv \bar{\alpha}^{\prime}+\left(\omega_{t}^{*}-\underline{\alpha}^{\prime}\right)-\omega_{t+1}^{*}$, which is a contradiction. Therefore, $\bar{\alpha}_{t}^{*} \geq \mathbf{0}$ must hold for all $t$.
} 
closed, we can define an implicit production function $\Gamma^{P}: \mathbb{R}_{+} \times \mathbb{R}_{+}^{n} \times \mathbb{R}_{+}^{n} \rightarrow$ $\mathbb{R}$ as:

$$
\begin{aligned}
& \left(-\alpha_{l},-\underline{\alpha}, \bar{\alpha}\right) \in P \Leftrightarrow \Gamma^{P}\left(\alpha_{l}, \underline{\alpha}, \bar{\alpha}\right) \leqq 0 ; \text { and } \\
& \left(-\alpha_{l},-\underline{\alpha}, \bar{\alpha}\right) \in \partial P \Leftrightarrow \Gamma^{P}\left(\alpha_{l}, \underline{\alpha}, \bar{\alpha}\right)=0 .
\end{aligned}
$$

This function is continuous and convex. Then, it is easy to show the existence of a feasible sequence $\left\{\left(c_{t}^{\prime}, \Lambda_{t}^{\prime}, \delta_{t}^{\prime}, \alpha_{t}^{\prime}, \omega_{t+1}^{\prime}\right)\right\}_{t=0}^{+\infty}$ such that for each $t, \underline{\alpha}_{t}^{\prime}+\delta_{t}^{\prime}<$ $\omega_{t}^{\prime}, \bar{\alpha}_{t}^{\prime}+\delta_{t}^{\prime}>c_{t}^{\prime}+\omega_{t+1}^{\prime}$, and $\Gamma^{P}\left(\alpha_{l t}^{\prime}, \underline{\alpha}_{t}^{\prime}, \bar{\alpha}_{t}^{\prime}\right)<0$, and therefore we can apply the Kuhn-Tucker Theorem. Thus, there exists a profile of non-zero and nonnegative Lagrange multipliers $\left(\mu_{l t}, \underline{\mu}, \bar{\mu}_{t}, \varsigma_{t}\right) \in \mathbb{R}_{+} \times \mathbb{R}_{+}^{n} \times \mathbb{R}_{+}^{n} \times \mathbb{R}_{++}$for each $t$ such that the non-trivial interior solution $\left\{\left(c_{t}^{*}, \Lambda_{t}^{*}, \delta_{t}^{*}, \alpha_{t}^{*}, \omega_{t+1}^{*}\right)\right\}_{t=0}^{+\infty}$ to $M P^{s p}$ is an optimal solution to the following maximisation programme $M P^{L}$ :

$$
\begin{aligned}
& \max _{\left\{\left(c_{t}, \Lambda_{t}, \delta_{t}, \alpha_{t}, \omega_{t+1}\right)\right\}_{t=0}^{+\infty}} \sum_{t=0}^{+\infty} \rho^{t}\left[v\left(c_{t}\right)+N \phi\left(L-\Lambda_{t}\right)-\mu_{l t}\left(\alpha_{l t}-N \Lambda_{t}\right)-\underline{\mu}_{t}\left(\underline{\alpha}_{t}+\delta_{t}-\omega_{t}\right)\right. \\
& \left.+\bar{\mu}_{t}\left(\bar{\alpha}_{t}+\delta_{t}-c_{t}-\omega_{t+1}\right)-\varsigma_{t} \Gamma^{P}\left(\alpha_{l t}, \underline{\alpha}_{t}, \bar{\alpha}_{t}\right)\right] .
\end{aligned}
$$

Note that $\varsigma_{t}>0$ must hold. Then, since $\Gamma^{P}$ is convex, there exists a suitable non-zero vector $\left(q_{l t}, \underline{q}_{t}, \bar{q}_{t}\right)$ for each $t$ such that

$$
\begin{aligned}
q_{l t} \alpha_{l t}^{*}+\underline{q}_{t} \underline{\alpha}_{t}^{*}+\bar{q}_{t} \bar{\alpha}_{t}^{*} & =0 ; \text { and } \\
q_{l t} \alpha_{l t}+\underline{q}_{t} \underline{\alpha}_{t}+\bar{q}_{t} \bar{\alpha}_{t} & \leqq 0 \text { for all }\left(-\alpha_{l},-\underline{\alpha}, \bar{\alpha}\right) \in P
\end{aligned}
$$

and moreover the following first order condition holds for $\left\{\left(c_{t}^{*}, \Lambda_{t}^{*}, \delta_{t}^{*}, \alpha_{t}^{*}, \omega_{t+1}^{*}\right)\right\}_{t=0}^{+\infty}$ : for each $t$,

$$
\begin{aligned}
\nabla v\left(c_{t}^{*}\right)-\bar{\mu}_{t} & =0, \\
-\phi^{\prime}\left(L-\Lambda_{t}^{*}\right)+\mu_{l t} & =0, \\
-\mu_{l t}-\varsigma_{t} q_{l t} & =0, \\
-\underline{\mu}_{t}-\varsigma_{t} \underline{q}_{t} & =0, \\
\bar{\mu}_{t}-\varsigma_{t} \bar{q}_{t} & =0, \\
-\bar{\mu}_{t}+\rho \underline{\mu}_{t+1} & =0, \\
\left(-\underline{\mu}_{t}+\bar{\mu}_{t}\right) \delta_{t}^{*} & =0 .
\end{aligned}
$$


From (A1), $\bar{\mu}_{t}=\nabla v\left(c_{t}^{*}\right)>\mathbf{0}$. From (A2), $\mu_{l t}=\phi^{\prime}\left(L-\Lambda_{t}^{*}\right)>0$. Then, by (A3) and $\varsigma_{t}>0, q_{l t}<0$. From (A5) and $\bar{\mu}_{t}>\mathbf{0}$, it follows that $\bar{q}_{t}>\mathbf{0}$. From (A6) and $\bar{\mu}_{t}>\mathbf{0}$, it follows that $\underline{\mu}_{t+1}>\mathbf{0}$. Then, by (A4), $\underline{q}_{t+1}<\mathbf{0}$. Moreover, from (A6) and (A4), $\bar{\mu}_{t}=-\rho \varsigma_{t+1} \underline{q}_{t+1}$, which in turn implies by (A5) that $\varsigma_{t} \bar{q}_{t}=-\rho \varsigma_{t+1} \underline{q}_{t+1}$.

Let $\left(w_{t}^{*}, p_{t}^{*}\right) \equiv\left(-q_{l t}, \bar{q}_{t}\right)$ for each $t$. Then, $\varsigma_{t} p_{t}^{*}=-\rho \varsigma_{t+1} \underline{q}_{t+1}$. Let $\left(1+r_{t+1}^{*}\right) \equiv \frac{\varsigma_{t}}{\varsigma_{t+1}} \rho^{-1}$. Then, $-\underline{q}_{t+1}=\left(1+r_{t+1}^{*}\right) p_{t}^{*}$ holds. In this case, $\varsigma_{t} p_{t}^{*}=\varsigma_{t+1} \rho\left(1+r_{t+1}^{*}\right) p_{t}^{*}$ holds, so that $\varsigma_{t}=\varsigma_{t+1} \rho\left(1+r_{t+1}^{*}\right)$. From (A1), (A2), (A3), and (A5), $\varsigma_{t}=\frac{\phi^{\prime}\left(L-\Lambda_{t}^{*}\right)}{w_{t}^{*}}=\frac{v_{i}^{\prime}\left(c_{t}^{*}\right)}{p_{i t}^{*}}$ holds for each commodity $i$ and each $t$. Therefore, $\varsigma_{t}=\varsigma_{t+1} \rho\left(1+r_{t+1}^{*}\right)$ implies the Euler equation

$$
\frac{\phi^{\prime}\left(L-\Lambda_{t}^{*}\right)}{w_{t}^{*}}=\rho\left(1+r_{t+1}^{*}\right) \frac{\phi^{\prime}\left(L-\Lambda_{t+1}^{*}\right)}{w_{t+1}^{*}} \text { for each } t .
$$

Finally, in (A7), note that $-\mu_{t}+\bar{\mu}_{t}=-\varsigma_{t}\left(1+r_{t}^{*}\right) p_{t-1}^{*}+\varsigma_{t} p_{t}^{*}$. To make $\delta_{t}^{*} \geqq \mathbf{0}$ for each $t$ the optimal solution to the Lagrangian maximization programme $M P^{L},-\left(1+r_{t}^{*}\right) p_{t-1}^{*}+p_{t}^{*} \leqq \mathbf{0}$ must hold. Thus, the non-arbitrage condition $\left(1+r_{t}\right) p_{t-1}^{*} \geqq p_{t}^{*}$ holds for each $t$.

In summary, we get a sequence of price vectors $\left(\boldsymbol{p}^{*}, \boldsymbol{w}^{*}, \boldsymbol{r}^{*}\right) \equiv\left\{\left(p_{t}^{*}, w_{t}^{*}, r_{t}^{*}\right)\right\}_{t=0}^{+\infty}$ such that $p_{t}^{*}>\mathbf{0}, w_{t}^{*}>0$, and

$$
\begin{aligned}
p_{t}^{*} \bar{\alpha}_{t}^{*} & =\left(1+r_{t}^{*}\right) p_{t-1}^{*} \underline{\alpha}_{t}^{*}+w_{t}^{*} \alpha_{l t}^{*} ; \text { and } \\
p_{t}^{*} \bar{\alpha}_{t} & \leqq\left(1+r_{t}^{*}\right) p_{t-1}^{*} \underline{\alpha}_{t}+w_{t}^{*} \alpha_{l t} \text { for any } \alpha_{t} \in P
\end{aligned}
$$

hold for each $t$. Then, given $\left(\boldsymbol{p}^{*}, \boldsymbol{w}^{*}, \boldsymbol{r}^{*}\right),\left\{\left(c_{t}^{*}, \Lambda_{t}^{*}, \delta_{t}^{*}, \alpha_{t}^{*}, p_{t-1}^{*} \omega_{t}^{*}, \omega_{t+1}^{*}\right)\right\}_{t=0}^{+\infty}$ constitutes an optimal solution to the following programme:

$$
\max _{\left\{\left(c_{t}, \Lambda_{t}, \delta_{t}, \beta_{t}, z_{t}, \omega_{t+1}\right)\right\}_{t=0}^{+\infty}} \sum_{t=0}^{+\infty} \rho^{t}\left(v\left(c_{t}\right)+N \phi\left(L-\Lambda_{t}\right)\right)
$$

subject to: for each $t$,

$$
\begin{aligned}
{\left[p_{t}^{*} \bar{\beta}_{t}-\left(1+r_{t}^{*}\right) p_{t-1}^{*} \underline{\beta}_{t}\right]+\left(1+r_{t}^{*}\right) z_{t}+p_{t}^{*} \delta_{t} } & =p_{t}^{*} c_{t}+p_{t}^{*} \omega_{t+1} \\
z_{t}+p_{t-1}^{*} \delta_{t} & =p_{t-1}^{*} \omega_{t} \\
p_{t-1}^{*} \underline{\beta}_{t} & =z_{t} \\
\beta_{t} & \in P, \Lambda_{t} \leqq L
\end{aligned}
$$


We can now show that $\left(\boldsymbol{p}^{*}, \boldsymbol{r}^{*}\right)$ can support an IRS. To see this, it is suffficient to note that $\left\{\left(c_{t}^{*}, \Lambda_{t}^{*}, \delta_{t}^{*}, \alpha_{t}^{*}, p_{t-1}^{*} \omega_{t}^{*}, \omega_{t+1}^{*}\right)\right\}_{t=0}^{+\infty}$ is consistent with each country's programme $M P^{\nu}$ given the price $\left(\boldsymbol{p}^{*}, \boldsymbol{r}^{*}\right)$. Given $\Omega_{0}$, let $\theta^{\nu} \equiv$ $\frac{p_{-1}^{*} \omega_{0}^{\nu}}{p_{-1}^{*} \omega_{0}}$ for each $\nu \in \mathcal{N}$. Then, for each $t$, let

$$
\gamma_{t}^{\nu} \equiv \frac{\frac{1}{N}\left[p_{t}^{*} \bar{\alpha}_{t}^{*}-\left(1+r_{t}^{*}\right) p_{t-1}^{*} \underline{\alpha}_{t}^{*}\right]+\theta^{\nu}\left[\left(1+r_{t}^{*}\right) p_{t-1}^{*} \omega_{t}-p_{t}^{*} \omega_{t+1}\right]}{\left[p_{t}^{*} \bar{\alpha}_{t}^{*}-\left(1+r_{t}^{*}\right) p_{t-1}^{*} \underline{\alpha}_{t}^{*}\right]+\left[\left(1+r_{t}^{*}\right) p_{t-1}^{*} \omega_{t}-p_{t}^{*} \omega_{t+1}\right]}>0 .
$$

By definition, for each $t, \sum_{\nu \in \mathcal{N}} \gamma_{t}^{\nu}=1$ holds. Then, for each $t$, let

$$
\left(c_{t}^{* \nu}, \Lambda_{t}^{* \nu}, \delta_{t}^{* \nu}, \beta_{t}^{* \nu}, z_{t}^{* \nu}, \omega_{t+1}^{* \nu}\right) \equiv\left(\gamma_{t}^{\nu} c_{t}^{*}, \Lambda_{t}^{*}, \theta^{\nu} \delta_{t}^{*}, \frac{\alpha_{t}^{*}}{N}, \theta^{\nu} p_{t-1}^{*} \omega_{t}^{*}, \theta^{\nu} \omega_{t+1}^{*}\right)
$$

for each $\nu \in \mathcal{N}$. Then, for each $\nu \in \mathcal{N}, \xi^{\nu} \equiv\left\{\left(c_{t}^{* \nu}, \Lambda_{t}^{* \nu}, \delta_{t}^{* \nu}, \beta_{t}^{* \nu}, z_{t}^{* \nu}, \omega_{t+1}^{* \nu}\right)\right\}_{t=0}^{+\infty}$ constitutes an optimal solution to the following programme $M P^{\nu}$ given $\left(\boldsymbol{p}^{*}, \boldsymbol{r}^{*}\right)$ :

$$
M P^{\nu}: \max _{\left\{\left(c_{t}^{\nu}, \Lambda_{t}^{\nu}, \delta_{t}^{\nu}, \beta_{t}^{\nu}, z_{t}^{\nu}, \omega_{t+1}^{\nu}\right)\right\}_{t=0}^{+\infty}} \sum_{t=0}^{+\infty} \rho^{t}\left(v\left(c_{t}^{\nu}\right)+\phi\left(L-\Lambda_{t}^{\nu}\right)\right)
$$

subject to: for every $t$,

$$
\begin{aligned}
{\left[p_{t}^{*} \bar{\beta}_{t}^{\nu}-\left(1+r_{t}^{*}\right) p_{t-1}^{*} \underline{\beta}_{t}^{\nu}\right]+\left(1+r_{t}^{*}\right) z_{t}^{\nu}+p_{t}^{*} \delta_{t}^{\nu} } & =p_{t}^{*} c_{t}^{\nu}+p_{t}^{*} \omega_{t+1}^{\nu} \\
z_{t}^{\nu}+p_{t-1}^{*} \delta_{t}^{\nu} & =p_{t-1}^{*} \omega_{t}^{\nu} ; \\
\beta_{t}^{\nu} & \in P, \Lambda_{t}^{\nu}=\beta_{l t}^{\nu} \leqq L .
\end{aligned}
$$

Thus, $\xi^{\nu} \in \mathcal{O}^{\nu}\left(\boldsymbol{p}^{*}, \boldsymbol{r}^{*}\right)$ holds for each $\nu \in \mathcal{N}$.

Moreover, by the definition, $\sum_{\nu \in \mathcal{N}} \bar{\beta}_{t}^{* \nu}=\sum_{\nu \in \mathcal{N}} c_{t}^{* \nu}+\sum_{\nu \in \mathcal{N}} \omega_{t+1}^{* \nu}$ and $\sum_{\nu \in \mathcal{N}} \underline{\beta}_{t}^{* \nu}=\sum_{\nu \in \mathcal{N}} \omega_{t}^{* \nu}$ hold for every $t$. Finally, $\sum_{\nu \in \mathcal{N}} p_{t-1}^{*} \underline{\beta}_{t}^{* \nu}=\sum_{\nu \in \mathcal{N}} z_{t}^{* \nu}$ holds for every $t$. In summary, $\left(\boldsymbol{p}^{*}, \boldsymbol{r}^{*}\right)$ associated with $\left(\xi^{\nu}\right)_{\nu \in \mathcal{N}}$ is an IRS.

\section{References}

[1] Aliprantis and Camera (2009): "A Correspondence-Theoretic Approach to Dynamic Optimization", Macroeconomic Dynamics 13 (Supplement 1), $97-117$. 